\title{
Finite model reasoning over existential rules
}

\author{
GIOVANNI AMENDOLA, NICOLA LEONE, MARCO MANNA \\ Department of Mathematics and Computer Science, University of Calabria, Italy \\ (e-mail: $\{$ amendola, leone, manna\}@mat.unical.it) \\ submitted 1 January 2003; revised 1 January 2003; accepted 1 January 2003
}

\begin{abstract}
Ontology-based query answering (OBQA) asks whether a Boolean conjunctive query is satisfied by all models of a logical theory consisting of a relational database paired with an ontology. The introduction of existential rules (i.e., Datalog rules extended with existential quantifiers in rule-heads) as a means to specify the ontology gave birth to Datalog+/-, a framework that has received increasing attention in the last decade, with focus also on decidability and finite controllability to support effective reasoning. Five basic decidable fragments have been singled out: linear, weakly-acyclic, guarded, sticky, and shy. Moreover, for all these fragments, except shy, the important property of finite controllability has been proved, ensuring that a query is satisfied by all models of the theory iff it is satisfied by all its finite models. In this paper we complete the picture by demonstrating that finite controllability of OBQA holds also for shy ontologies, and it therefore applies to all basic decidable Datalog+/- classes. To make the demonstration, we devise a general technique to facilitate the process of (dis)proving finite controllability of an arbitrary ontological fragment.
\end{abstract}

KEYWORDS: Existential rules, Datalog, Finite controllability, Finite model reasoning, Query answering.

\section{Introduction}

The problem of answering a Boolean query $q$ against a logical theory consisting of an extensional database $D$ paired with an ontology $\Sigma$ is attracting the increasing attention of scientists in various fields of Computer Science, ranging from Artificial Intelligence (Baget et al. 2011, Calvanese et al. 2013; Gottlob et al. 2014) to Database Theory (Bienvenu et al. 2014, Gottlob et al. 2014 Bourhis et al. 2016) and Logic (Pérez-Urbina et al. 2010, Bárány et al. 2014, Gottlob et al. 2013). This problem, called ontology-based query answering, for short OBQA (Calì et al. 2009b), is usually stated as $D \cup \Sigma \models q$, and it is equivalent to checking whether $q$ is satisfied by all models of $D \cup \Sigma$ according to the standard approach of first-order logics, yielding an open world semantics.

Description Logics (Baader et al. 2003) and Datalog ${ }^{ \pm}$(Calì et al. 2009a) have been recognized as the two main families of formal knowledge representation languages to specify $\Sigma$, while union of (Boolean) conjunctive queries, $\mathrm{U}(\mathrm{B}) \mathrm{CQs}$ for short, is the most common and studied formalism to express $q$. For both these families, OBQA is generally undecidable (Rosati 2007; Calì et al. 2013). Hence, a number of syntactic decidable fragments of the above ontological languages have been singled out. However, decidability alone is not the only desideratum. For example, a good balance between computational complexity and expressive power is, without any doubt, of high importance too. But there is another property that is turning out to be as interesting as challenging to prove: it goes under the name of finite controllability (Rosati 2006). An ontological fragment $\mathcal{F}$ is finitely controllable if, for each triple $\langle D, \Sigma, q\rangle$ with $\Sigma \in \mathcal{F}$, it holds 


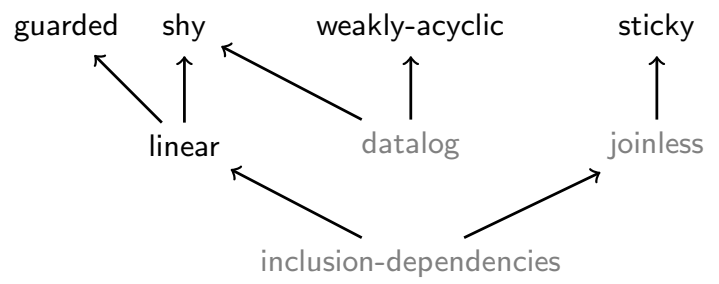

Fig. 1. Taxonomy of the basic Datalog ${ }^{ \pm}$classes.

that $D \cup \Sigma \not \models q$ implies that there is a finite model $M$ of $D \cup \Sigma$ such that $M \not \forall q$. This is usually stated as $D \cup \Sigma \models q$ if, and only if, $D \cup \Sigma \models_{\text {fin }} q$ (where $\models_{\text {fin }}$ stands for entailment under finite models), as the "only if" direction is always trivially true. And there are contexts, like in databases (Johnson and Klug 1984, Rosati 2006; Bárány et al. 2014), in which reasoning with respect to finite models is preferred.

In this paper we focus on the Datalog ${ }^{ \pm}$family, which has been introduced with the aim of "closing the gap between the Semantic Web and databases" (Calì et al. 2012) to provide the Web of Data with scalable formalisms that can benefit from existing database technologies. In fact, Datalog $^{ \pm}$generalizes two well-known subfamilies of Description Logics called $\mathcal{E} \mathcal{L}$ and DL-Lite, which collect the basic tractable languages for OBQA in the context of the Semantic Web and databases. In particular, we consider ontologies where $\Sigma$ is a set of existential rules, each of which is a first-order formula $\rho$ of the form $\forall \mathbf{X} \forall \mathbf{Y}(\phi(\mathbf{X}, \mathbf{Y}) \rightarrow \exists \mathbf{Z} p(\mathbf{X}, \mathbf{Z}))$, where the body $\phi(\mathbf{X}, \mathbf{Y})$ of $\rho$ is a conjunction of atoms, and the head $p(\mathbf{X}, \mathbf{Z})$ of $\rho$ is a single atom.

The main decidable Datalog ${ }^{ \pm}$fragments rely on the following five syntactic properties: weakacyclicity (Fagin et al. 2005), guardedness (Calì et al. 2013), linearity (Calì et al. 2012), stickiness (Calì et al. 2010), and shyness (Leone et al. 2012). And these properties underlie the basic classes called weakly-acyclic, guarded, linear, sticky, and shy, respectively. Several variants and combinations of these classes have been defined and studied too (Baget et al. 2010, Krötzsch and Rudolph 2011, Calì et al. 2012, Civili and Rosati 2012; Gottlob et al. 2013), as well as semantic properties subsuming the syntactic ones (Baget et al. 2009, Leone et al. 2012).

The five basic classes above are pairwise uncomparable, except for linear which is strictly contained in both guarded and shy, as depicted in Figure 1 Interestingly, both weakly-acyclic and shy strictly contain datalog — the well-known class with rules of the form $\forall \mathbf{X} \forall \mathbf{Y}(\phi(\mathbf{X}, \mathbf{Y}) \rightarrow p(\mathbf{X}))$, where existential quantification has been dropped. Moreover, sticky strictly contains joinless - the class collecting sets of rules where each body contains no repeated variable. The latter, introduced by Gogacz and Marcinkowski (2013) to prove that sticky is finitely controllable, plays a central role also in this paper. Finally, both linear and joinless strictly contain inclusiondependencies - the well-known class of relational database dependencies collecting sets of rules with one single body atom and no repeated variable.

Under arbitrary models, OBQA can be reduced to the problem of answering $q$ over a universal (or canonical) model $U$ that can be homomorphically embedded into every other model (both finite and infinite) of $D \cup \Sigma$. Therefore, $D \cup \Sigma \models q$ if, and only if, $U \models q$. A way to compute a universal model is to employ the so called chase procedure. Starting from $D$, the chase "repairs" violations of rules by repeatedly adding new atoms —introducing fresh values, called nulls, whenever required by an existential variable - until a fixed-point satisfying all rules is reached. In the classical setting, the chase is therefore sound and complete. But when finite model reason- 
ing (namely reasoning over finite models only, here denoted by $\models_{\text {fin }}$ ) is required, then the chase is generally uncomplete, unless ontologies are finitely controllable. Hence, proving this property is of utmost importance, especially in those contexts where finite model reasoning is relevant.

Finite controllability of weakly-acyclic comes for free since every ontology here admits a finite universal model, computed by a variant of the chase procedure which goes under the name of restricted chase (Fagin et al. 2005). Conversely, the proof of this property for the subsequent three classes has been a very different matter. Complex, yet intriguing, constructions have been devised for linear (Rosati 2006, Bárány et al. 2014), guarded (Bárány et al. 2014), and more recently for sticky (Gogacz and Marcinkowski 2013). To complete the picture, we have addressed the same problem for shy and get the following positive result, which is the main contribution of the paper.

\section{Theorem 1.1}

Under shy ontologies, $D \cup \Sigma \models q$ if, and only if, $D \cup \Sigma \models_{\text {fin }} q$.

For the proof, we design in Section 3 and exploit in Section 4 a general technique (our second contribution), called canonical rewriting, to facilitate the process of (dis)proving finite controllability of an arbitrary ontological fragment of existential rules. By exploiting this technique, we can immediately (re)confirm that linear is finitely controllable since inclusion-dependencies is. In addition, we prove (our third contribution) that sticky-join (Calì et al. 2012), generalizing both sticky and linear, is finitely controllable since sticky is. However, differently from linear and sticky-join, the canonical rewriting of a shy ontology - although it is simpler and still a shy ontology - does not immediately fall in any other known class. Therefore, to prove that shy is finitely controllable, we devise three technical tools on top of the canonical rewriting from which we are able to exploit the fact that joinless is finitely controllable.

\section{Ontology-based query answering}

Basics. Let $\mathbf{C}, \mathbf{N}$ and $\mathbf{V}$ denote pairwise disjoint discrete sets of constants, nulls and variables, respectively. An element $t$ of $\mathbf{T}=\mathbf{C} \cup \mathbf{N} \cup \mathbf{V}$ is called term. An atom $\alpha$ is a labeled tuple $p\left(t_{1}, \ldots, t_{m}\right)$, where $p$ is a predicate symbol, $m$ is the arity of both $p$ anf $\alpha$, and $t_{1}, \ldots, t_{m}$ are terms. An atom is simple if it contains no repeated term. We denote by $\operatorname{pred}(\alpha)$ the predicate symbol $p$, and by $\alpha[i]$ the $i$-th term $t_{i}$ of the $\alpha$. We also consider propositional atoms, which are simple atoms of arity 0 written without brackets. Given two sets $A$ and $B$ of atoms, a homomorphism from $A$ to $B$ is a mapping $h: \mathbf{T} \rightarrow \mathbf{T}$ such that $c \in \mathbf{C}$ implies $h(c)=c$, and also $p\left(t_{1}, \ldots, t_{m}\right) \in A$ implies $p\left(h\left(t_{1}\right), \ldots, h\left(t_{m}\right)\right) \in B$. As usual, we denote by $h(A)=\left\{p\left(h\left(t_{1}\right), \ldots, h\left(t_{m}\right)\right): p\left(t_{1}, \ldots, t_{m}\right) \in\right.$ $A\} \subseteq B$. An instance $I$ is a discrete set of atoms where each term is either a constant or a null.

Syntax. A database $D$ is a finite null-free instance. An (existential) rule $\rho$ is a first-order formula $\forall \mathbf{X} \forall \mathbf{Y}(\phi(\mathbf{X}, \mathbf{Y}) \rightarrow \exists \mathbf{Z} p(\mathbf{X}, \mathbf{Z}))$, where $\operatorname{body}(\rho)=\phi(\mathbf{X}, \mathbf{Y})$ is a conjunction of atoms, and head $(\rho)=p(\mathbf{X}, \mathbf{Z})$ is an atom. Constants may occur in $\rho$. If $\mathbf{Z}=\emptyset$, then $\rho$ is datalog rule. An ontology $\Sigma$ is a set of rules. For each rule $\rho$ of $\Sigma$, we denote by $\mathbf{V}(\rho)$ the set of variables appearing in $\rho$, by $\mathbf{E V}(\rho)$ the set of all existential variables of $\rho$, and by $\mathbf{U V}(\rho)$ the set of all universal variables of $\rho$. A union of Boolean conjunctive query, UBCQ for short, $q$ is a firstorder expression of the form $\exists \mathbf{Y}_{1} \psi_{1}\left(\mathbf{Y}_{1}\right) \vee \ldots \vee \exists \mathbf{Y}_{k} \psi_{k}\left(\mathbf{Y}_{k}\right)$, where each $\psi_{j}\left(\mathbf{Y}_{j}\right)$ is a conjunction of atoms. Constants may occur also in $q$. In case $k=1$, then $q$ is simply called BCQ.

Semantics. Consider a triple $\langle D, \Sigma, q\rangle$ as above. An instance I satisfies a rule $\rho \in \Sigma$, denoted by $I \models \rho$, if whenever there is a homomorphism $h$ from $\operatorname{bod} y(\rho)$ to $I$, then there is a homomorphism 
$\left.h^{\prime} \supseteq h\right|_{\mathbf{X}}$ from $\{h e a d(\rho)\}$ to $I$. Moreover, I satisfies $\Sigma$, denoted by $I \models \Sigma$, if $I$ satisfies each rule of $\Sigma$. The models of $D \cup \Sigma$, denoted by $\operatorname{mods}(D, \Sigma)$, consist of the set $\{I: I \supseteq D$ and $I \models \Sigma\}$. An instance $I$ satisfies $q$, written $I \models q$, if there is a homomorphism from some $\psi_{j}\left(\mathbf{Y}_{j}\right)$ to $I$. Also, $q$ is true over $D \cup \Sigma$, written $D \cup \Sigma \models q$, if each model of $D \cup \Sigma$ satisfies $q$.

The chase. Consider a logical theory $\langle D, \Sigma\rangle$ as above. A rule $\rho$ of $\Sigma$ is applicable to an instance $I$ if there is a homomorphism $h$ from $\operatorname{bod} y(\rho)$ to $I$ that maps the existential variables of $\rho$ to different nulls not occurring in $I$. If so, $\langle\rho, h\rangle(I)=I \cup h($ head $(\rho))$ defines a chase step. The chase procedure (Deutsch et al. 2008) of $D \cup \Sigma$ is any sequence $I_{0}=D \subset I_{1} \subset \ldots \subset I_{m} \subset \ldots$ of instances obtained by applying exhaustively the rules of $\Sigma$ in a fair (e.g., breadth-first) fashion in such a way that, for each $i>0,\langle\rho, h\rangle\left(I_{i-1}\right)=I_{i}$ defines a chase step for some $\rho$ and $h$. We call chase $(D, \Sigma)$ the (possibly infinite) instance $\bigcup_{i \geqslant 0} I_{i}$. Importantly, different chase steps introduce different nulls. This variant of the chase is called oblivious, and defines a family of isomorphic instances, namely any two such instances are equal modulo renaming of nulls. Hence, without loss of generality, it is common practice to consider the oblivious chase as deterministic and its least fixpoint as unique. The restricted version of this procedure imposes a further condition on each chase step: $I \not \models h^{\prime}(\operatorname{head}(\rho))$, where $h^{\prime}=\left.h\right|_{\mathbf{U V}(\rho)}$. Differently from the oblivious one, it defines a family of homomorphically equivalent instances, each generically denoted by rchase $(D, \Sigma)$. It is well-known that $(r)$ chase $(D, \Sigma)$ is a universal model of $D \cup \Sigma$, namely for each $M \in \operatorname{mods}(D, \Sigma)$, there is a homomorphism from $\operatorname{chase}(D, \Sigma)$ to $M$. Hence, given a UBCQ $q$, it holds that $(r) \operatorname{chase}(D, \Sigma) \models q$ if, and only if, $D \cup \Sigma \models q($ Fagin et al. 2005).

Finite controllability. The finite models of a theory $D \cup \Sigma$, denoted by $\operatorname{fmods}(D, \Sigma)$, are the finite instances in $\{I \in \operatorname{mods}(D, \Sigma):|I| \in \mathbb{N}\}$. An ontological fragment $\mathcal{F}$ is finitely controllable if, for each database $D$, for each ontology $\Sigma$ of $\mathcal{F}$, and for each UBCQ $q$, it holds that $D \cup \Sigma \not \models q$ implies that there exists a finite model $M$ of $D \cup \Sigma$ such that $M \not \forall q$. This is formally stated as $D \cup \Sigma \models q$ if and only if $D \cup \Sigma \models_{\text {fin }} q$, or equivalently chase $(D, \Sigma) \models q$ if and only if $D \cup \Sigma \models_{\text {fin }} q$.

\subsection{Datalog $^{ \pm}$fragments}

Fix a database $D$, an ontology $\Sigma$, and a chase step involving some pair $\langle\bar{\rho}, h\rangle$. To lighten the presentation, we assume that different rules of $\Sigma$ share no variable. Also, for every $m$-ary predicate $p$ and every $i \in\{1, \ldots, m\}$, the pair $(p, i)$ is called position and denoted by $p[i]$. Finally, given a set $A$ of atoms, a term $t$ occurs in $A$ at position $p[i]$ if there is $\alpha \in A$ s.t. $\operatorname{pred}(\alpha)=p$ and $\alpha[i]=t$.

Local conditions. $\Sigma$ belongs to: $(i)$ datalog whenever $\rho \in \Sigma$ implies $\mathbf{E V}(\rho)=\emptyset$; (ii) inclusiondependencies whenever $\rho \in \Sigma$ implies that $\rho$ contains only simple atoms and $|\operatorname{body}(\rho)|=1$; (iii) linear whenever $\rho \in \Sigma$ implies $|\operatorname{body}(\rho)|=1$; (iv) guarded whenever $\rho \in \Sigma$ implies that there is an atom of $\operatorname{bod} y(\rho)$ containing all the variables of $\mathbf{U V}(\rho) ;(v)$ joinless whenever $\rho \in \Sigma$ implies that head $(\rho)$ is a simple atom and $\operatorname{body}(\rho)$ contains no repeated variables.

Weak-acyclicity (Fagin et al. 2005). Informally, $\Sigma \in$ weakly-acyclic guarantees that: if $X$ occurs in $\operatorname{body}(\bar{\rho})$ at position $p[i]$ and $h(X) \in \mathbf{N}$, then the number of distinct nulls occurring in rchase $(D, \Sigma)$ at position $p[i]$ are finitely many. Formally, the labeled graph $G(\Sigma)$ associated to $\Sigma$ is defined as the pair $\langle N, A\rangle$, where $(i) N$ collects all the positions $p[1], \ldots, p[m]$ for each $m$-ary predicate $p$ occurring in $\Sigma$; (ii) $(p[i], r[j]$,plain $) \in A$ if there is a rule $\rho \in \Sigma$ and a variable $X$ of $\rho$ such that: $X$ occurs in the body of $\rho$ at position $p[i]$ and $X$ occurs in the head of $\rho$ at position $r[j]$; and $(i i i)(p[i], r[j]$, special $) \in A$ if there is a rule $\rho \in \Sigma$, a universal variable $X$ occurring also in the head of $\rho$, and an existential variable $Z$ of $\rho$ such that: $X$ occurs in the body of $\rho$ at 

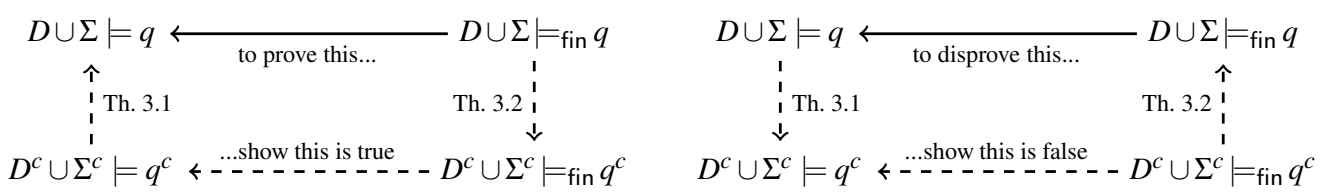

Fig. 2. Application of the canonical rewriting.

position $p[i]$ and $Z$ occurs in the head of $\rho$ at position $r[j]$. Ontology $\Sigma$ belongs to weakly-acyclic if $G(\Sigma)$ has no cycle going through an arc labeled as special.

Stickiness (Cali et al. 2012). Informally, $\Sigma \in$ sticky guarantees that: if $X$ occurs multiple times in $\operatorname{body}(\bar{\rho})$, then $X$ occurs in head $(\bar{\rho})$ and $h(X)$ belongs to every atom of chase $(D, \Sigma)$ that depends on $h($ head $(\bar{\rho}))$. Formally, a variable $X$ of $\Sigma$ is marked if $(i)$ there is a rule $\rho \in \Sigma$ such that $X$ occurs in $\operatorname{body}(\rho)$ but not in head $(\rho)$; or $(i i)$ there are two rules $\rho, \rho^{\prime} \in \Sigma$ such that a marked variable occurs in body $(\rho)$ at some position $p[i]$ and $X$ occurs in head $\left(\rho^{\prime}\right)$ at position $p[i]$ too. Ontology $\Sigma$ belongs to sticky if, for each $\rho \in \Sigma$, the following condition is satisfied: if $X$ occurs multiple times in $\operatorname{bod} y(\rho)$, then $X$ is not marked. A more refined condition identifies interesting cases in which it is safe to allow rules containing some marked variable that occurs multiple times but in a single body atom only. This refinement gives rise to sticky-join, generalizing both sticky and linear.

Shyness (Leone et al. 2012). Informally, $\Sigma \in$ shy guarantees that: (1) if $X$ occurs in two different atoms of $\operatorname{bod} y(\bar{\rho})$, then $h(X) \in \mathbf{C}$; and (2) if $X$ and $Y$ occur both in head $(\bar{\rho})$ and in two different atoms of body $(\bar{\rho})$, then $h(X)=h(Y)$ implies $h(X) \in \mathbf{C}$. Formally, consider an existential variable $X$ of $\Sigma$. Position $p[i]$ is invaded by $X$ if there is a rule $\rho$ of $\Sigma$ such that: $(i) X$ occurs in head $(\rho)$ at position $p[i]$, or (ii) some universal variable $Y$ of $\rho$ is attacked by $X$-namely $Y$ occurs in $\operatorname{body}(\rho)$ only at positions invaded by $X$ - and it also occurs in head $(\rho)$ at position $p[i]$. A universal variable is protected if it is attacked by no existential variable. Ontology $\Sigma$ belongs to shy if, for each $\rho \in \Sigma$, the following conditions are both satisfied: (1) if $X$ occurs in two different atoms of $\operatorname{body}(\rho)$, then $X$ is protected; and (2) if $X$ and $Y$ occur both in head $(\rho)$ and in two different atoms of $\operatorname{bod} y(\rho)$, then $X$ and $Y$ are not attacked by the same variable.

\section{Canonical rewriting}

In this section we design a general technique to facilitate the process of (dis)proving finite controllability of an arbitrary ontological fragment of existential rules. More specifically, from a triple $\langle D, \Sigma, q\rangle$ we build the triple $\left\langle D^{c}, \Sigma^{c}, q^{c}\right\rangle$ enjoying the following properties: (1) $D^{c}$ is propositional database; (2) $\Sigma^{c}$ are constant-free rules containing only simple atoms; (3) $q^{c}$ is a constant-free UBCQ with only simple atoms; $(4) \operatorname{chase}\left(D^{c}, \Sigma^{c}\right)$ is a constant-free instance containing only simple atoms; and (5) there is a "semantic" correspondence between $\operatorname{mods}(D, \Sigma)$ and $\bmod s\left(D^{c}, \Sigma^{c}\right)$. By exploiting these properties, one can apply the technique shown in Figure 2

\subsection{Overview}

Consider the database $D=\{$ person(tim), person(john), fatherOf(tim,john $)\}$, and the ontology $\Sigma=\{\operatorname{person}(X) \rightarrow \exists Y$ fatherOf $(Y, X) ;$ fatherOf $(X, Y) \rightarrow \operatorname{person}(X)\}$. Let $p, f, c_{1}$ and $c_{2}$ be shorthands of person, fatherOf, tim and john, respectively. Hence, chase $(D, \Sigma)$ is the instance 
$D \cup\left\{f\left(n_{1}, c_{1}\right), f\left(n_{2}, c_{2}\right)\right\} \cup\left\{p\left(n_{i}\right), f\left(n_{i+2}, n_{i}\right)\right\}_{i>0}$, where each $n_{i}$ denotes a distinct null of $\mathbf{N}$. From $D$ we construct the propositional database $D^{c}=\left\{p_{\left[c_{1}\right]}, p_{\left[c_{2}\right]}, f_{\left[c_{1}, c_{2}\right]}\right\}$ obtained by encoding in the predicates the tuples of $D$. Then, from $\Sigma$ we construct $\Sigma^{c}$ collecting the following rules:

$$
\begin{array}{rllr}
p_{\left[c_{1}\right]} \rightarrow \exists Y f_{\left[1, c_{1}\right]}(Y) & f_{\left[c_{1}, c_{1}\right]} \rightarrow p_{\left[c_{1}\right]} & f_{\left[c_{1}, 1\right]}(Y) \rightarrow p_{\left[c_{1}\right]} & f_{[1,1]}(X) \rightarrow p_{[1]}(X) \\
p_{\left[c_{2}\right]} \rightarrow \exists Y f_{\left[1, c_{2}\right]}(Y) & f_{\left[c_{1}, c_{2}\right]} \rightarrow p_{\left[c_{1}\right]} & f_{\left[c_{2}, 1\right]}(Y) \rightarrow p_{\left[c_{2}\right]} & f_{[1,2]}(X, Y) \rightarrow p_{[1]}(X) \\
p_{[1]}(X) & \rightarrow \exists Y f_{[1,2]}(Y, X) & f_{\left[c_{2}, c_{1}\right]} \rightarrow p_{\left[c_{2}\right]} & f_{\left[1, c_{1}\right]}(X) \rightarrow p_{[1]}(X) \\
& f_{\left[c_{2}, c_{2}\right]} \rightarrow p_{\left[c_{2}\right]} & f_{\left[1, c_{2}\right]}(X) \rightarrow p_{[1]}(X) &
\end{array}
$$

The predicates here encode tuples of terms consisting of database constants $\left(c_{1}\right.$ and $\left.c_{2}\right)$ and placeholders of nulls (1 and 2). Consider the first rule $\rho=p(X) \rightarrow \exists Y f(Y, X)$ applied by the chase over $D \cup \Sigma$, and $h=\left\{X \mapsto c_{1}, Y \mapsto n_{1}\right\}$ be its associated homomorphism. Hence, $h(\operatorname{bod} y(\rho))=p\left(c_{1}\right)$ and $h(h e a d(\rho))=f\left(n_{1}, c_{1}\right)$. Such an application is mimed by the "sister" rule $\rho^{c}=p_{\left[c_{1}\right]} \rightarrow$ $\exists Y f_{\left[1, c_{1}\right]}(Y)$. By exploiting the same homomorphism we obtain $h\left(\operatorname{body}\left(\rho^{c}\right)\right)=p_{\left[c_{1}\right]}$ and also $h\left(\right.$ head $\left.\left(\rho^{c}\right)\right)=f_{\left[1, c_{1}\right]}\left(n_{1}\right)$. Actually, the encoded tuple $\left[c_{1}\right]$ in $p_{\left[c_{1}\right]}$ says that the original twin atom $p\left(c_{1}\right)$ is unary and its unique term is exactly $c_{1}$. Moreover, the encoded tuple $\left[1, c_{1}\right]$ in $f_{\left[1, c_{1}\right]}\left(n_{1}\right)$ says that the original twin atom $f\left(n_{1}, c_{1}\right)$ is binary, that its first term is a null, and that its second term is exactly the constant $c_{1}$. Since from predicate $f_{\left[1, c_{1}\right]}$ we only know that the first term is a null, it must be unary to keep the specific null value. In the above construction, red rules are those applied by the chase on $D^{c} \cup \Sigma^{c}$. For example, rule $f_{\left[1, c_{1}\right]}(X) \rightarrow p_{[1]}(X)$ mimics $f(X, Y) \rightarrow p(X)$ when $X$ is mapped to a null and $Y$ to $c_{1}$; and rule $f_{[1,2]}(X, Y) \rightarrow p_{[1]}(X)$ mimics $f(X, Y) \rightarrow p(X)$ when $X$ and $Y$ are mapped to different nulls. Hence, chase $\left(D^{c}, \Sigma^{c}\right)$ is:

$$
D^{c} \cup\left\{p_{[1]}\left(n_{i}\right)\right\}_{i>0} \cup\left\{f_{\left[1, c_{1}\right]}\left(n_{1}\right), f_{\left[1, c_{2}\right]}\left(n_{2}\right)\right\} \cup\left\{f_{[1,2]}\left(n_{i+2}, n_{i}\right)\right\}_{i>0} .
$$

As a result, the rewriting separates the interaction between the database constants propagated body-to-head via universal variables and the nulls introduced to satisfy existential variables. Also, since the predicates encode the "shapes" of the twin atoms — namely $f_{[1,2]}(X, Y)$ means different nulls while $f_{[1,1]}(X)$ the same null- repeated variables are encoded too. By following the same approach, we can rewrite also the query. Consider for example the BCQ $q=\exists X p(X), f\left(X, c_{1}\right)$. Therefore, $q^{c}$ is the UBCQ: $\left(p_{\left[c_{1}\right]}, f_{\left[c_{1}, c_{1}\right]}\right) \vee\left(p_{\left[c_{2}\right]}, f_{\left[c_{2}, c_{1}\right]}\right) \vee\left(\exists X p_{[1]}(X), f_{\left[1, c_{1}\right]}(X)\right)$.

\subsection{Formal construction and properties}

Let us fix a triple $\langle D, \Sigma, q\rangle$ through the rest of this section. Consider an atom $\alpha=p\left(t_{1}, \ldots, t_{m}\right)$ with terms over $\mathbf{C} \cup \mathbf{V}$. The canonical atom of $\alpha$ is the atom $\alpha^{c}=p_{\left[\ell_{1}, \ldots, \ell_{m}\right]}\left(\tau_{1}, \ldots, \tau_{\mu}\right)$, where: (a) $\ell_{i}=t_{i}$ if $t_{i} \in \mathbf{C}$; (b) $\ell_{i}=\ell_{j}$ if $t_{i}=t_{j}$; or $(c) \ell_{i}=1+\max \left(\{0\} \cup\left\{\ell_{j} \in \mathbb{N}: j<i\right\}\right)$ if $t_{i} \in \mathbf{V}$ and $t_{j} \neq t_{i} \forall j<i$ and $\tau_{i}=V \in \mathbf{V}$, if there exists $t_{j}$ such that $\ell_{j}=i$ and $t_{j}=V$. Moreover, given a set of atoms $A$, we define $A^{c}=\left\{\alpha^{c}: \alpha \in A\right\}$, and give a rule $\rho$, we define $\rho^{c}$ as the rule so that $\operatorname{bod} y\left(\rho^{c}\right)=\operatorname{body}(\rho)^{c}$ and head $\left(\rho^{c}\right)=h e a d(\rho)^{c}$. For instance, let $\alpha=p\left(c_{1}, X, c_{2}, X, Y, Z, Y\right)$ be an atom. Then, the canonical atom $\alpha^{c}$ of $\alpha$ is given by $p_{\left[c_{1}, 1, c_{2}, 1,2,3,2\right]}(X, Y, Z)$. Note that, by definition of $\tau_{i}$, for $i=1, \ldots, \mu$, we have that the arity $\mu \leq m$ of the canonical atom is equal to $\max \left(\{0\} \cup\left\{f\left(t_{j}\right) \in \mathbb{N}: j \leq m\right\}\right)$.

Definition 3.1 (Safe and Canonical substitutions)

A map $\varsigma: \operatorname{const}(D \cup \Sigma) \cup \mathbf{V} \rightarrow$ const $(D \cup \Sigma) \cup \mathbf{V}$ is called canonical substitution if $\varsigma(c)=c$ for each $c \in \operatorname{const}(D \cup \Sigma)$. Moreover, we say that a canonical substitution $\varsigma$ is safe w.r.t. a rule $\rho \in \Sigma$ if $\varsigma(\mathbf{U V}(\rho)) \subseteq \operatorname{const}(D \cup \Sigma) \cup \mathbf{U V}(\rho)$, and $\varsigma(V)=V$, for each $V \in \mathbf{E V}(\rho)$. 
Intuitively, a safe substitution maps each existential variable to itself and no universal variable is mapped to an existental one. As usual, given a set of atoms $A$, we denote by $\varsigma(A)=$ $\left\{p\left(\varsigma\left(t_{1}\right), \ldots, \varsigma\left(t_{m}\right)\right): p\left(t_{1}, \ldots, t_{m}\right)\right\}$, and given a rule $\rho$, we denote by $\varsigma(\rho)$ the rule such that $\operatorname{body}(\varsigma(\rho))=\varsigma(\operatorname{body}(\rho))$ and head $(\varsigma(\rho))=\varsigma(\operatorname{head}(\rho))$.

Example 3.1

Consider $D=\left\{r\left(c_{1}, c_{3}\right)\right\}$ and $\Sigma$ consisting of the following rules: $\rho_{1}=r\left(Y_{1}, Z_{1}\right), p\left(W_{1}, X_{1}, X_{1}, Y_{1}\right)$ $\rightarrow \exists T_{1} g\left(X_{1}, Y_{1}, T_{1}, X_{1}, Z_{1}\right)$ and $\rho_{2}=s\left(X_{2}\right), t\left(Y_{2}\right) \rightarrow r\left(X_{2}, Y_{2}\right)$. For instance, $\varsigma_{1}=\left\{c_{1} \mapsto c_{1}, c_{3} \mapsto\right.$ $\left.c_{3}, Y_{1} \mapsto X_{1}, Z_{1} \mapsto c_{3}, W_{1} \mapsto Y_{1}, X_{1} \mapsto X_{1}, T_{1} \mapsto T_{1}\right\}$ and $\varsigma_{1}^{\prime}=\left\{c_{1} \mapsto c_{1}, c_{3} \mapsto c_{3}, Y_{1} \mapsto c_{1}, Z_{1} \mapsto X_{1}\right.$, $\left.W_{1} \mapsto c_{1}, X_{1} \mapsto Y_{1}, T_{1} \mapsto T_{1}\right\}$ are safe substitutions w.r.t. $\rho_{1}$. Indeed, const $(D \cup \Sigma)=\left\{c_{1}, c_{3}\right\}$, $\mathbf{U V}\left(\rho_{1}\right)=\left\{W_{1}, X_{1}, Y_{1}, Z_{1}\right\}, \mathbf{E V}\left(\rho_{1}\right)=\left\{T_{1}\right\}$, the existential variable $T_{1}$ is mapped to itself, and no other variable is mapped to an existential one. Moreover, $\varsigma_{1}\left(\rho_{1}\right)=r\left(X_{1}, c_{3}\right), p\left(Y_{1}, X_{1}, X_{1}, X_{1}\right) \rightarrow$ $\exists T_{1} g\left(X_{1}, X_{1}, T_{1}, X_{1}, c_{3}\right)$ and $\zeta_{1}^{\prime}\left(\rho_{1}\right)=r\left(c_{1}, X_{1}\right), p\left(c_{1}, Y_{1}, Y_{1}, c_{1}\right) \rightarrow \exists T_{1} g\left(Y_{1}, c_{1}, T_{1}, Y_{1}, X_{1}\right)$.

We denote by $\mathcal{C S}$ the set of all canonical substitutions and by $\beta(\rho) \subseteq \mathcal{C S}$ the set of all safe substitutions w.r.t. $\rho$. Given a set of atoms $A$ [resp. a rule $\rho$ ] and a canonical substitution [resp. safe substitution] $\varsigma$, we say that $\varsigma(A)^{c}$ [resp. $\varsigma(\rho)^{c}$ ] is the canonical set of atoms w.r.t $A$ [resp. canonical rule w.r.t. $\rho$ ] and $\varsigma$. Observe that two different canonical substitutions could produce two isomorphic canonical set of atoms. For instance, let $A=\{p(X, Y)\}$, and consider $\varsigma=\{X \mapsto X, Y \mapsto Y\}$ and $\varsigma^{\prime}=\{X \mapsto Y, Y \mapsto X\}$. Then, $\varsigma(A)^{c}=\left\{p_{[1,2]}(X, Y)\right\}$, and $\varsigma^{\prime}(A)^{c}=\left\{p_{[1,2]}(Y, X)\right\}$ are isomorphic set of atoms. Therefore, to avoid redundancies, we denote by $\mathcal{C S}^{*}\left[\right.$ resp. $\left.\beta^{*}(\rho)\right]$ any arbitrary maximal subset of $\mathcal{C S}$ [resp. of $\left.\beta(\rho)\right]$ producing canonical set of atoms [resp. canonical rules] containing no two isomorphic elements.

We denote by $\Sigma^{c}$ the set of all canonical rules $\left\{\varsigma(\rho)^{c}: \rho \in \Sigma\right.$ and $\left.\varsigma \in \beta^{*}(\rho)\right\}$, and we call it the canonical rewriting of $\Sigma$. Also, given a UBCQ $q$ of the form $\exists \mathbf{Y}_{1} \psi_{1}\left(\mathbf{Y}_{1}\right) \vee \ldots \vee \exists \mathbf{Y}_{k} \psi_{k}\left(\mathbf{Y}_{k}\right)$, we denote by $q^{c}$ the disjunction $\bigvee_{\varsigma_{1} \in \mathcal{C} \mathcal{S}^{*}} \varsigma_{1}\left(\psi_{1}\left(\mathbf{Y}_{1}\right)\right)^{c} \vee \ldots \vee \bigvee_{\varsigma_{k} \in \mathcal{C} \mathcal{S}^{*}} \varsigma_{k}\left(\psi_{k}\left(\mathbf{Y}_{k}\right)\right)^{c}$. and we call it the canonical rewriting of $q$. Finally, we call $D^{c}$ the canonical rewriting of $D$.

\section{Proposition 3.1}

The triple $\left\langle D^{c}, \Sigma^{c}, q^{c}\right\rangle$ can be constructed from $\langle D, \Sigma, q\rangle$ in polynomial time (in data complexity).

Example 3.2

Consider the ontology $\Sigma$ with the safe substitutions $\varsigma_{1}$ and $\varsigma_{1}^{\prime}$ w.r.t. $\rho_{1}$ of the Example 3.1 Therefore, we obtain the canonical rules: $\varsigma_{1}\left(\rho_{1}\right)^{c}=r_{\left[1, c_{3}\right]}\left(X_{1}\right), p_{[1,2,2,2]}\left(Y_{1}, X_{1}\right) \rightarrow \exists T_{1} g_{\left[1,1,2,1, c_{3}\right]}\left(X_{1}, T_{1}\right)$ and $\varsigma_{1}^{\prime}\left(\rho_{1}\right)^{c}=r_{\left[c_{1}, 1\right]}\left(X_{1}\right), p_{\left[c_{1}, 1,1, c_{1}\right]}\left(Y_{1}\right) \rightarrow \exists T_{1} g_{\left[1, c_{1}, 2,1,3\right]}\left(Y_{1}, T_{1}, X_{1}\right)$. Moreover, let $\varsigma_{2}$ and $\varsigma_{2}^{\prime}$ be the safe substitutions containing $\left\{X_{2} \mapsto X_{2}, Y_{2} \mapsto X_{2}\right\}$ and $\left\{X_{2} \mapsto c_{1}, Y_{2} \mapsto c_{3}\right\}$ w.r.t. $\rho_{2}$, respectively. Hence, we have $\varsigma_{2}\left(\rho_{2}\right)^{c}=s_{[1]}\left(X_{2}\right), t_{[1]}\left(X_{2}\right) \rightarrow r_{[1,1]}\left(X_{2}\right)$ and $\varsigma_{2}^{\prime}\left(\rho_{2}\right)^{c}=s_{\left[c_{1}\right]}, t_{\left[c_{3}\right]} \rightarrow r_{\left[c_{1}, c_{3}\right]}$. Therefore, $\varsigma_{1}\left(\rho_{1}\right)^{c}, \varsigma_{1}^{\prime}\left(\rho_{1}\right)^{c}, \varsigma_{2}\left(\rho_{2}\right)^{c}$, and $\varsigma_{2}^{\prime}\left(\rho_{2}\right)^{c}$ are (some of the) rules of $\Sigma^{c}$.

We consider a function $\mathfrak{R}$ from the set of atoms of $D^{c} \cup \Sigma^{c}$ to the set of atom of $D \cup \Sigma$. For each atom $\alpha=a_{\left[s_{1}, \ldots, s_{m}\right]}\left(\sigma_{1}, \ldots, \sigma_{\mu}\right)$, we build an atom $\mathfrak{R}(\alpha)=a\left(t_{1}, \ldots, t_{m}\right)$ such that: $(a) t_{i}=s_{i}$ if $s_{i} \in \mathbf{C} ;(b) t_{i}=\sigma_{i}$ if $s_{i}=k$ and $s_{j} \neq k$, for each $j<i$; or $(c) t_{i}=\sigma_{j}$ if $s_{i}=s_{j}$, for some $j<i$.

For instance, let $\alpha=p_{\left[1, c_{1}, 2,1, c_{2}, 1,2\right]}(X, Y)$ be an atom of the logical theory $D^{c} \cup \Sigma^{c}$. Then, $\mathfrak{R}(\alpha)=p\left(X, c_{1}, Y, X, c_{2}, X, Y\right)$. We call $\mathfrak{R}$ the unpacking function. Given a set of atoms $A$ of $D^{c} \cup \Sigma^{c}$, we denote by $\mathfrak{R}(A)=\{\mathfrak{R}(\alpha): \alpha \in A\}$ the corresponding set of atoms of $D \cup \Sigma$. If $I$ is an instance, we call $\mathfrak{R}(I)$ the unpacked instance of $I$. Given a rule $\rho^{c}$ in $\Sigma^{c}$, we denote by $\mathfrak{R}\left(\rho^{c}\right)$ the rule obtained applying $\mathfrak{R}$ to each atom in $\rho^{c}$, i.e. $\mathfrak{R}\left(\rho^{c}\right): \mathfrak{R}\left(\operatorname{bod} y\left(\rho^{c}\right)\right) \rightarrow \mathfrak{R}\left(\right.$ head $\left.\left(\rho^{c}\right)\right)$, and we call it the unpacked rule of $\rho^{c}$. Similarly, we denote by $\mathfrak{R}\left(q^{c}\right)$ the query obtained applying $\mathfrak{R}$ 
to the atoms of the UBCQ $q^{c}$, and we call it the unpacked query of $q^{c}$. Informally, the unpacking function acts as the inverse operator to the canonical rewriting. Moreover, it enjoys an interesting and useful property: the chase of a logical theory coincides with the unpacking of the chase constructed from of the same theory given in canonical form:

\section{Proposition 3.2}

Consider a set $\Sigma$ of existential rules. For each database $D$ and for each UBCQ $q$, it holds that $\mathfrak{R}\left(\operatorname{chase}\left(D^{c}, \Sigma^{c}\right)\right)=\operatorname{chase}(D, \Sigma)$ and $\mathfrak{R}\left(q^{c}\right) \equiv q$.

By exploiting the above proposition, we can now prove that a UBCQ $q$ is satisfied by all models of a theory $D \cup \Sigma$ if, and only if, each model of the canonical rewriting of the theory $D^{c} \cup \Sigma^{c}$ satisfies the canonical rewriting of the UBCQ $q^{c}$.

Theorem 3.1

$D \cup \Sigma \models q$ if, and only if, $D^{c} \cup \Sigma^{c} \models q^{c}$.

Note that, if $\Sigma$ is a constant-free ontology, then, for each model $M^{c}$ of $D^{c} \cup \Sigma^{c}, \mathfrak{R}\left(M^{c}\right)$ is a model of $D \cup \Sigma$. The request for a constant-free ontology is needed. Indeed, for instance, let $\Sigma=\{p(a) \rightarrow r(a) ; r(x) \rightarrow p(x)\}$. So that, $\Sigma^{c}=\left\{p_{[a]} \rightarrow r_{[a]} ; r_{[a]} \rightarrow p_{[a]} ; r_{[1]}\left(V_{1}\right) \rightarrow p_{[1]}\left(V_{1}\right)\right\}$. Therefore, $M^{c}=\left\{p_{[1]}(a)\right\}$ is a model of $\Sigma^{c}$, but $\mathfrak{R}\left(M^{c}\right)=\{p(a)\}$ is not a model of $\Sigma$, as it does not satisfy the first rule. However, we can overcome this problem considering the following class of models. Given a model $M^{c} \in \bmod s\left(D^{c}, \Sigma^{c}\right)$, we say that $N^{c}$ is a smooth instance of $M^{c}$ if there exists a bijective map $f$ :terms $\left(M^{c}\right) \rightarrow \operatorname{terms}\left(N^{c}\right)$ such that $f(n)=n$ for each null $n \in \operatorname{terms}\left(M^{c}\right)$; $f(c)=n_{c}$ for each constant $c \in \operatorname{terms}\left(M^{c}\right)$, where $n_{c}$ is a fresh null; and $f\left(M^{c}\right)=N^{c}$. Note that a smooth instance of a model $M^{c}$ is also a model of $D^{c} \cup \Sigma^{c}$ and it is also constant-free.

Proposition 3.3

If $M^{c} \in \operatorname{mods}\left(D^{c}, \Sigma^{c}\right)$, then $\mathfrak{R}\left(N^{c}\right) \in \bmod s(D, \Sigma)$, for each smooth model $N^{c}$ of $M^{c}$.

By exploiting the above proposition, we can now prove that a UBCQ $q$ is satisfied by all finite models of a theory $D \cup \Sigma$ if, and only if, each finite model of the canonical rewriting of the theory $D^{c} \cup \Sigma^{c}$ satisfies the canonical rewriting of the UBCQ $q^{c}$.

Theorem 3.2

$D \cup \Sigma \models_{\text {fin }} q$ if, and only if, $D^{c} \cup \Sigma^{c} \models_{\text {fin }} q^{c}$.

Proof

Assume that $D \cup \Sigma \models_{\text {fin }} q$. Then, for each finite model $M$ of $D \cup \Sigma$, there exists a homomorphism $h$ from at least one disjunct of $q$, say $\psi_{j}\left(\mathbf{Y}_{j}\right)$ to $M$. Now, let $M^{c}$ be a finite model of $D^{c} \cup \Sigma^{c}$. By Proposition 3.3, there exist a (finite) smooth model $N^{c} \in \operatorname{mods}\left(D^{c}, \Sigma^{c}\right)$ of $M^{c}$ and a bijective map $f$ from terms $\left(M^{c}\right)$ to terms $\left(N^{c}\right)$ such that $f(n)=n$ for each null $n \in \operatorname{terms}\left(M^{c}\right) ; f(c)=n_{c}$ for each constant $c \in \operatorname{terms}\left(M^{c}\right)$, where $n_{c}$ is a fresh null; $f\left(M^{c}\right)=N^{c}$, and $\mathfrak{R}\left(N^{c}\right) \in \operatorname{mods}(D, \Sigma)$. Hence, by assumption, there exists a homomorphism $h$ from some $\psi_{j}\left(\mathbf{Y}_{j}\right)$ to $\Re\left(N^{c}\right)$. Let $A=$ $h\left(\psi_{j}\left(\mathbf{Y}_{j}\right)\right) \subseteq \Re\left(N^{c}\right)$. Then, for each atom $\alpha \in A$, we can choose an arbitrary atom $\beta \in N^{c}$ such that $\mathfrak{R}(\beta)=\alpha$. Let $B$ such a subset of $N^{c}$. Therefore, by construction, there exists a BCQ in $q^{c}$ isomorphic to $B$. In particular, there exists a homomorphism $h$ from $q^{c}$ to $N^{c}$. In conclusion, $f^{-1} \circ h$ is a homomorphism from $q^{c}$ to $M^{c}$. Indeed, $f^{-1} \circ h$ is a map from $\operatorname{terms}\left(q^{c}\right)$ to terms $\left(M^{c}\right)$ such that $f^{-1}\left(h\left(q^{c}\right)\right) \subseteq f^{-1}\left(N^{c}\right)=M^{c}$. Now, assume that $D^{c} \cup \Sigma^{c} \models_{\text {fin }} q^{c}$. Let $M$ be a finite model of $D \cup \Sigma$. By definition of canonical rules, can be easily proved that there exists a finite model 


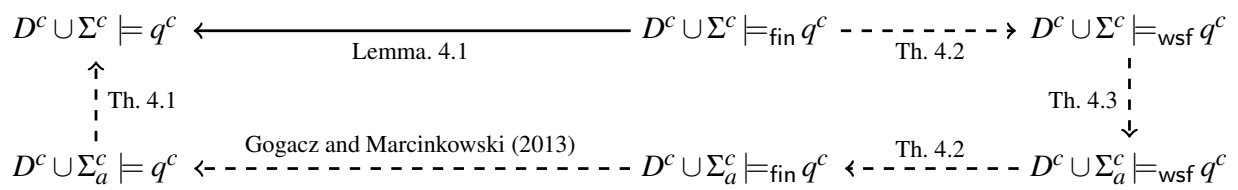

Fig. 3. Chain of implications for the proof of Lemma 4.1.

$M^{c} \in \operatorname{fmods}\left(D^{c}, \Sigma^{c}\right)$ such that $\mathfrak{R}\left(M^{c}\right)=M$. Hence, let $h$ be a homomorphism from $q^{c}$ to $M^{c}$. So that, $h\left(\varsigma_{j}\left(\psi_{j}\left(\mathbf{Y}_{j}\right)\right)^{c}\right) \subseteq M^{c}$, for some disjunct $\varsigma_{j}\left(\psi_{j}\left(\mathbf{Y}_{j}\right)\right)^{c}$ of $q^{c}$. Therefore, by applying the unpacked function, we have that $\mathfrak{R}\left(h\left(\varsigma_{j}\left(\psi_{j}\left(\mathbf{Y}_{j}\right)\right)^{c}\right)\right)=h\left(\Re\left(\varsigma_{j}\left(\psi_{j}\left(\mathbf{Y}_{j}\right)\right)^{c}\right)\right)=h\left(\varsigma_{j}\left(\psi_{j}\left(\mathbf{Y}_{j}\right)\right) \subseteq\right.$ $\mathfrak{R}\left(M^{c}\right)=M$. Hence, $h$ is also a homomorphism from $q$ to $M$.

\subsection{Immediate consequences}

By exploiting the properties of the canonical rewriting, one can reprove that linear is finitely controllable, and prove (for the first time) that also sticky-join enjoys this property. In fact, given a linear or sticky-join ontology $\Sigma$, its canonical rewriting $\Sigma^{c}$ belongs to inclusion-dependencies or sticky, respectively. In the former case, it suffices to observe that any variable occurring multiple times in some atom $\alpha$, by definition, occurs exactly once in its associated canonical atom $\alpha^{c}$. In the latter case, additionally, consider a variable $X$ violating the sticky property since it is marked and it occurs multiple times in the body of some rule $\rho$. By hypothesis, $X$ may occur in exactly one atom of $\operatorname{bod} y(\rho)$. However, even if marked, $X$ now occurs exactly once in its canonical atom and it cannot violate the sticky property any more. The following result follows.

Theorem 3.3

Under sticky-join ontologies, $D \cup \Sigma \models q$ if, and only if, $D \cup \Sigma \models_{\text {fin }} q$.

\section{Finite controllability of Shy ontologies}

We open this section by observing that, differently from linear and sticky-join, the canonical rewriting of a shy ontology —although it is still a shy ontology — does not fall in any other known class. To prove that shy is finitely controllable, we therefore devise three technical tools on top of the canonical rewriting defined in Section 3 These tools allow us to show that $D^{c} \cup \Sigma^{c} \models_{\text {fin }} q^{c}$ if, and only if, $D^{c} \cup \Sigma^{c} \models q^{c}$ (Lemma 4.1). To this end, let us fix a triple $\langle D, \Sigma, q\rangle$, and the associated one $\left\langle D^{c}, \Sigma^{c}, q^{c}\right\rangle$ in canonical form. Our tools are as follows:

Active and harmless rules. Whenever $\Sigma$ is shy, we can partition $\Sigma^{c}$ in two sets, denoted by $\Sigma_{a}^{c}$ and $\Sigma_{h}^{c}$ —collecting active and harmless rules, respectively — enjoying the following properties: (1) $\Sigma_{h}^{c}$ are the rules of $\Sigma^{c}$ with at least a variable occurring in more than one body atom; (2) $\Sigma_{a}^{c}=\Sigma^{c} \backslash \Sigma_{h}^{c}$ is a joinless (and still shy) ontology; and (3) $\operatorname{chase}\left(D^{c}, \Sigma^{c}\right)=\operatorname{chase}\left(D^{c}, \Sigma_{a}^{c}\right)$.

Well-supported finite models. Inspired by well-supported interpretations of general logic programs (Fages 1991), we define well-supported finite models of $\langle D, \Sigma\rangle$, denoted by wsfmods $(D, \Sigma)$, which enjoy the following properties: (1) for each $M \in$ wsfmods $(D, \Sigma)$, there exists an ordering $\left(\alpha_{1}, \ldots, \alpha_{m}\right)$ of its atoms such that, for each $\alpha_{j}$ of $M$, either $\alpha_{j}$ belongs to $D$, or there exist a rule $\rho \in \Sigma$ and a homomorphism from the atoms of $\rho$ to $\left\{\alpha_{1}, \ldots, \alpha_{j}\right\}$ that maps $\operatorname{body}(\rho)$ to 
$\left\{\alpha_{1}, \ldots, \alpha_{j-1}\right\}$ and $\operatorname{head}(\rho)$ to $\left\{\alpha_{j}\right\} ;(2)$ for each $M \in \operatorname{fmods}(D, \Sigma)$, there exists a well-supported finite model $M^{\prime} \subseteq M$; and (3) each minimal finite model of $D \cup \Sigma$ is a well-supported finite model.

Propagation ordering. Since $\bmod \left(D^{c}, \Sigma^{c}\right) \subseteq \bmod s\left(D^{c}, \Sigma_{a}^{c}\right)$, in general it is definitely possible that a model $M$ of $D^{c} \cup \Sigma_{a}^{c}$ is not a model of $D^{c} \cup \Sigma^{c}$. In case $\Sigma$ is shy and $M$ is a well-supported finite models of $D^{c} \cup \Sigma_{a}^{c}$, by exploiting an arbitrary ordering of $M$, we show how to rename and propagate some of the terms of $M$ to construct an instance $M^{\prime}$ enjoying the following property: (1) $M^{\prime} \in$ wsfmods $\left(D^{c}, \Sigma^{c}\right)$; and (2) there exists a homomorphism from $M^{\prime}$ to $M$.

With these tools in place, we can now apply the technique shown in Figure 3, where we use the symbol $\models_{\text {wsf }}$ to refer the satisfiability of the query under well-supported finite models only.

\subsection{Active and harmless rules}

As said, and next stated, the canonical rewriting of a shy ontology is again a shy ontology.

Proposition 4.1

If $\Sigma$ is shy, then $\Sigma^{c}$ is.

The goal of this section is therefore to identify a suitable subset of $\Sigma^{c}$ that falls in some known finitely-controllable class, and that roughly "behaves" as $\Sigma^{c}$ under both finite and arbitrary models. The idea is to collect in $\Sigma_{h}^{c}$ the rules of $\Sigma^{c}$ with at least a variable occurring in more than one body atom, and to define $\Sigma_{a}^{c}=\Sigma^{c} \backslash \Sigma_{h}^{c}$. In other words, $\Sigma_{a}^{c}$ is exactly the maximal subset of $\Sigma^{c}$ that belongs to joinless. Let us now provide some insights regarding this way of partitioning $\Sigma^{c}$. From the database $D=\{p(c)\}$ and the shy ontology $\Sigma=\{p(X) \rightarrow \exists Y f(Y, X) ; f(X, Y), p(X) \rightarrow p(Y)\}$ we first construct $D^{c}=\left\{p_{[c]}\right\}$ and $\Sigma^{c}$ as the following set of rules:

$$
\begin{aligned}
& f_{[1, c]}(X), p_{[1]}(X) \rightarrow p_{[c]} \quad f_{[c, c]}, p_{[c]} \rightarrow p_{[c]} \quad p_{[c]} \rightarrow \exists Y f_{[1, c]}(Y) \\
& f_{[1,1]}(X), p_{[1]}(X) \rightarrow p_{[1]}(X) \quad f_{[c, 1]}(Y), p_{[c]} \rightarrow p_{[1]}(Y) \quad p_{[1]}(X) \rightarrow \exists Y f_{[1,2]}(Y, X) \\
& f_{[1,2]}(X, Y), p_{[1]}(X) \rightarrow p_{[1]}(Y)
\end{aligned}
$$

Again, red rules are those applied by the chase on $D^{c} \cup \Sigma^{c}$. Now we observe that there is no way to trigger the rules in the first column: although the chase does produce an atom $f\left(t, n_{i}\right)$ for some term $t$ and null $n_{i}$, it never produces any atom $p\left(n_{i}\right)$. This fact is detected by the syntactic conditions underlying shy (marking $X$ in $f(X, Y), p(X) \rightarrow p(Y)$ as "protected"), which guarantee that $X$ may be mapped by the chase to constants only. Hence, since by definition $\Sigma_{a}^{c}$ consists of the joinless rules in the last two columns, it holds that $\operatorname{chase}\left(D^{c}, \Sigma^{c}\right)=\operatorname{chase}\left(D^{c}, \Sigma_{a}^{c}\right)$.

The reason underlying the fact that the chase never applies rules of $\Sigma_{h}^{c}$ will be exploited in Section 4.3 to prove Theorem 4.3 (see Figure 3 , right-hand side), namely that $\Sigma_{a}^{c}$ roughly "behaves" as $\Sigma^{c}$ under finite (well-supported) models. Conversely, to show Theorem 4.1 (see Figure 3 , lefthand side) it suffices to observe the more general property that $\Sigma_{a}^{c} \subseteq \Sigma^{c}$, which immediately implies $\bmod s\left(D^{c}, \Sigma^{c}\right) \subseteq \bmod s\left(D^{c}, \Sigma_{a}^{c}\right)$. And the next result follows.

Theorem 4.1

If $D^{c} \cup \Sigma_{a}^{c} \models q^{c}$, then $D^{c} \cup \Sigma^{c} \models q^{c}$.

\subsection{Well-supported finite models}

We start by defining the notion of well-supported finite instances, which is inspired by the related notion of well-supported interpretations for general logic programs (Fages 1991). 
Let $D$ be a database, and $\Sigma$ be an ontology. A finite instance $I$ is called well-supported w.r.t. the theory $D \cup \Sigma$ if there is an ordering $\left(\alpha_{1}, \ldots, \alpha_{m}\right)$ of its atoms such that, for each $j \in\{1, \ldots, m\}$, at least one of the following conditions is satisfied: (1) $\alpha_{j}$ is a database atom of $D$; and (2) there exist a rule $\rho$ of $\Sigma$ and a homomorphism $h$ from atoms $(\rho)$ to $\left\{\alpha_{1}, \ldots, \alpha_{j}\right\}$ such that $h($ head $(\rho))=$ $\left\{\alpha_{j}\right\}$ and $h(\operatorname{bod} y(\rho)) \subseteq\left\{\alpha_{1}, \ldots, \alpha_{j-1}\right\}$. In both cases, we will say that $\alpha_{j}$ is a well-supported atom w.r.t. $\left(\alpha_{1}, \ldots, \alpha_{m}\right)$; while in the latter case we will also say that $\rho$ is a well-supporting rule for $\alpha_{j}$ w.r.t. $\left(\alpha_{1}, \ldots, \alpha_{m}\right)$. Such an ordering will be called a well-supported ordering of $I$.

We denote by $w \operatorname{smods}(D, \Sigma) \subseteq \operatorname{fmods}(D, \Sigma)$ the set of all well-supported finite models of $D \cup \Sigma$. Moreover, if a UBCQ $q$ is satisfied by each model of $w \operatorname{sfmod} s(D, \Sigma)$, we write $D \cup \Sigma \models$ wsf $q$. Interestingly, each finite model of $D \cup \Sigma$ contains a well-supported finite model of the theory.

\section{Proposition 4.2}

For each $M \in \operatorname{fmods}(D, \Sigma)$, there exists $M^{\prime} \subseteq M$ such that $M^{\prime} \in w \operatorname{sfmods}(D, \Sigma)$. In particular, each minimal finite model of $D \cup \Sigma$ is a well-supported finite model.

Although each finite model of an ontological theory contains a well-supported finite model of the theory, the reverse inclusion does not hold. Consider for example the ontology $\Sigma$ of Section 3.1, and the model $M=D \cup\left\{f\left(c_{1}, c_{1}\right), f\left(c_{2}, c_{1}\right)\right\}$. Since $\left(p\left(c_{1}\right), p\left(c_{2}\right), f\left(c_{1}, c_{2}\right), f\left(c_{1}, c_{1}\right)\right.$, $\left.f\left(c_{2}, c_{1}\right)\right)$ is a well-supported ordering of $M$, then $M$ is well-supported. However, $M \backslash\left\{f\left(c_{2}, c_{1}\right)\right\}$ is a model of $D \cup \Sigma$. Therefore, $M$ is not a minimal one. Using Proposition 4.2 we can now prove that if a UBCQ $q$ can be satisfied by each well-supported finite model of a theory, then it can be satisfied by each finite model of the theory.

Theorem 4.2

$D \cup \Sigma \models_{\text {wsf }} q$ if, and only if, $D \cup \Sigma \models_{\text {fin }} q$.

\section{Proof}

Clearly, by subset inclusion, if each finite model of $D \cup \Sigma$ satisfies the query $q$, then each wellsupported finite model of $D \cup \Sigma$ satisfies $q$. Moreover, as each finite minimal model is a wellsupported finite model (Proposition 4.2), then for each finite model $M^{\prime}$ of $D \cup \Sigma$, we can find a well-supported finite model, that is minimal, $M$ of $D \cup \Sigma$, such that $M \subseteq M^{\prime}$, and, in particular, there exists a homomorphism $h$ (i.e., the identity homomorphism) such that $h(M) \subseteq M^{\prime}$.

\subsection{Propagation ordering}

Let us start with the preliminary notions of existentially well-supported atom and propagated term. Let $I$ be a well-supported finite instance, and $\left(\alpha_{1}, \ldots, \alpha_{m}\right)$ be a well-supported ordering of $I$. An atom $\alpha$ of $I \backslash D$ is said existentially well-supported w.r.t. the ordering $\left(\alpha_{1}, \ldots, \alpha_{m}\right)$ if, for each well-supporting rule $\rho$ for $\alpha$ w.r.t. $\left(\alpha_{1}, \ldots, \alpha_{m}\right)$, it holds that $\mathbf{E V}(\rho) \neq \emptyset$. Moreover, let $\alpha_{j}[k]=t$, for some position $k$, then $t$ is said propagated from an atom $\alpha_{i}$ in position $l$, whenever $i<j, \alpha_{i}[l]=t$, and there exist a well-supporting rule $\rho$ for $\alpha_{j}$ and a homomorphism $h$ such that $\alpha_{i} \in h(\operatorname{bod} y(\rho))$. Consider again ontology $\Sigma$ of Section 3.1, and the well-supported finite model $M$ considered after Proposition 4.2. For instance, the atom $f\left(c_{1}, c_{1}\right)$ is existentially wellsupported. Indeed, the unique way to well-support the atom comes from the first rule of $\Sigma$, that is an existential rule. We are now ready to define the notion of propagation ordering. 
Definition 4.1 (Propagation ordering)

Let $D$ be a database, $\Sigma$ be a joinless ontology, $M \in$ wsfmods $(D, \Sigma)$, and $\left(\alpha_{1}, \ldots, \alpha_{m}\right)$ be a wellsupported ordering of $M$. For each $\alpha_{j} \in M$, we build a new atom $\left\langle\alpha_{j}\right\rangle$ as follows. Let $t=\alpha_{j}[k]$. We have: (1) If $\alpha_{j}$ is an existentially well-supported atom and $k$ is an existential position, then $\left\langle\alpha_{j}\right\rangle[k]=\langle t, j, k\rangle$, where $\langle t, j, k\rangle$ is called a starting point of $t$; (2) If $t$ is a propagated term from some atom $\alpha_{i}$ in position $l$, then $\left\langle\alpha_{j}\right\rangle[k]=\left\langle\alpha_{i}\right\rangle[l]$; and $(3)\left\langle\alpha_{j}\right\rangle[k]=\alpha_{j}[k]$, otherwise. We call $\left(\left\langle\alpha_{1}\right\rangle, \ldots,\left\langle\alpha_{m}\right\rangle\right)$ a propagation ordering of the well-supported ordering $\left(\alpha_{1}, \ldots, \alpha_{m}\right)$.

Note that the same term could have several starting points. This propagation ordering will be useful to remember a starting point of that particular term and its propagations in other atoms.

\section{Example 4.1}

Consider the following joinless ontology $\Sigma=\left\{s\left(X_{1}\right) \rightarrow \exists Y_{1} p\left(X_{1}, Y_{1}\right) ; s\left(X_{2}\right) \rightarrow \exists Y_{2} u\left(Y_{2}, X_{2}\right)\right.$; $\left.p\left(X_{3}, Y_{3}\right), u\left(W_{3}, Z_{3}\right) \rightarrow r\left(Y_{3}, Z_{3}\right) ; p\left(X_{4}, Y_{4}\right) \rightarrow t\left(Y_{4}\right)\right\}$, and the database $D=\left\{s\left(c_{1}\right)\right\}$. As example, $M=\left\{s\left(c_{1}\right), t\left(c_{2}\right), t\left(n_{1}\right), p\left(c_{1}, c_{2}\right), p\left(c_{1}, n_{1}\right), r\left(c_{2}, c_{1}\right), r\left(n_{1}, c_{1}\right), u\left(c_{2}, c_{1}\right), u\left(n_{1}, c_{1}\right)\right\}$ is a wellsupported finite model of $D \cup \Sigma$. Indeed, for instance, $\left(s\left(c_{1}\right), p\left(c_{1}, c_{2}\right), p\left(c_{1}, n_{1}\right), u\left(c_{2}, c_{1}\right), t\left(c_{2}\right)\right.$, $\left.u\left(n_{1}, c_{1}\right), r\left(n_{1}, c_{1}\right), t\left(n_{1}\right), r\left(c_{2}, c_{1}\right)\right)$ is a well-supported ordering of $M$. The existentially wellsupported atoms are $p\left(c_{1}, c_{2}\right), p\left(c_{1}, n_{1}\right), u\left(c_{2}, c_{1}\right)$ and $u\left(n_{1}, c_{1}\right)$. More specifically, $p\left(c_{1}, c_{2}\right)$ has the term $c_{2}$ in the existential position 2, then $\left\langle p\left(c_{1}, c_{2}\right)\right\rangle=p\left(c_{1},\left\langle c_{2}, 2,2\right\rangle\right)$, as $p\left(c_{1}, c_{2}\right)$ is the second atom of the well-supported ordering considered; $p\left(c_{1}, n_{1}\right)$ has the term $n_{1}$ in the existential position 2 , then $\left\langle p\left(c_{1}, n_{1}\right)\right\rangle=p\left(c_{1},\left\langle n_{1}, 3,2\right\rangle\right) ; u\left(c_{2}, c_{1}\right)$ has the term $c_{2}$ in the existential position 1 , then $\left\langle u\left(c_{2}, c_{1}\right)\right\rangle=u\left(\left\langle c_{2}, 4,1\right\rangle, c_{1}\right) ; u\left(n_{1}, c_{1}\right)$ has the term $n_{1}$ in the existential position 1 , then $\left\langle u\left(n_{1}, c_{1}\right)\right\rangle=u\left(\left\langle n_{1}, 6,1\right\rangle, c_{1}\right)$. On the other hand, the term $c_{2}$ is propagated in the atom $t\left(c_{2}\right)$ in the first (and unique) position. It comes from atom $p\left(c_{1}, c_{2}\right)$, and we know that the starting point of $c_{2}$ is $\left\langle c_{2}, 2,2\right\rangle$. Therefore, $\left\langle t\left(c_{2}\right)\right\rangle=t\left(\left\langle c_{2}, 2,2\right\rangle\right)$. Moreover, in a similarly way, we obtain that $\left\langle t\left(n_{1}\right)\right\rangle=t\left(\left\langle n_{1}, 3,2\right\rangle\right)$. Finally, the term $n_{1}$ is propagated in the atom $r\left(n_{1}, c_{1}\right)$ in the first position, and it comes from atom $p\left(c_{1}, n_{1}\right)$; whereas the term $c_{1}$ is propagated in the atom $r\left(n_{1}, c_{1}\right)$ in the second position, and it comes from atom $u\left(n_{1}, c_{1}\right)$. Therefore, $\left\langle r\left(n_{1}, c_{1}\right)\right\rangle=r\left(\left\langle n_{1}, 3,2\right\rangle, c_{1}\right)$. $\triangleleft$

With our technical tools in place, we are now able to prove the following technical result.

Theorem 4.3

For each $\Sigma \in$ shy, if $D^{c} \cup \Sigma^{c} \models_{\text {wsf }} q^{c}$ then $D^{c} \cup \Sigma_{a}^{c} \models_{\text {wsf }} q^{c}$.

\section{Proof intuition}

Consider an arbitrary model $M \in$ wsfmods $\left(D^{c}, \Sigma_{a}^{c}\right)$. It suffices to prove that there exist $M^{\prime} \in$ wsfmods $\left(D^{c}, \Sigma^{c}\right)$ and a homomorphism $h^{\prime}$ s.t. $h^{\prime}\left(M^{\prime}\right) \subseteq M$. Indeed, by hypothesis, there exists a homomorphism $h$ s.t. $h(q) \subseteq M^{\prime}$, and so $\left(h^{\prime} \circ h\right)(q) \subseteq M$.

The difficulty here is that $M$ could not be a model of $D^{c} \cup \Sigma^{c}$. Consider the database $D=\{s(c)\}$ and the shy ontology $\Sigma=\{s(X) \rightarrow \exists Y p(Y) ; s(X) \rightarrow \exists Y r(Y) ; p(X), r(X) \rightarrow g(X)\}$. The canonical rewriting is $D^{c}=\left\{s_{[c]}\right\}$ and $\Sigma^{c}$ as follows:

$$
\begin{aligned}
s_{[c]} & \rightarrow \exists Y p_{[1]}(Y) & s_{[c]} & \rightarrow \exists Y r_{[1]}(Y) & p_{[c]}, r_{[c]} & \rightarrow g_{[c]} \\
s_{[1]}(X) & \rightarrow \exists Y p_{[1]}(Y) & s_{[1]}(X) & \rightarrow \exists Y r_{[1]}(Y) & p_{[1]}(X), r_{[1]}(X) & \rightarrow g_{[1]}(X)
\end{aligned}
$$

One can verify that $M=\left\{s_{[c]}, p_{[1]}\left(n_{1}\right), r_{[1]}\left(n_{1}\right)\right\}$ is a (minimal) well-supported finite model of $D^{c} \cup \Sigma_{a}^{c}$ since, by Proposition 4.1. $\Sigma^{c}$ is shy, and since $\Sigma_{a}^{c}$ is obtained from $\Sigma^{c}$ by discarding the last harmless rule. However, $M$ is not a model of $D^{c} \cup \Sigma^{c}$ because the last rule is not satisfied. 
The idea is to show how to construct from $M$ a model $M^{\prime} \in w s f m o d s\left(D^{c}, \Sigma^{c}\right)$ that can be homomorphically mapped to $M$. Intuitively, we identify the starting points in which existential variables of $\Sigma_{a}^{c}$ have been satisfied and rename the introduced terms using a propagation ordering.

In the example above, consider the well-supported ordering $\left(s_{[c]}, p_{[1]}\left(n_{1}\right), r_{[1]}\left(n_{1}\right)\right)$ of $M$, replace $n_{1}$ in $p_{[1]}\left(n_{1}\right)$ by $\left\langle n_{1}, 2,2\right\rangle$ (null $n_{1}$ introduced in the second atom in the second position), and replace $n_{1}$ in $r_{[1]}\left(n_{1}\right)$ by $\left\langle n_{1}, 3,2\right\rangle$ (null $n_{1}$ introduced in the third atom in the second position). Then, since $M$ is well-supported, we propagate (if needed) these new terms according the supporting ordering. In our case, $M^{\prime}=\left\{s_{[c]}, p_{[1]}\left(\left\langle n_{1}, 2,2\right\rangle\right), r_{[1]}\left(\left\langle n_{1}, 3,2\right\rangle\right)\right\}$ is now a finite model of $D^{c} \cup \Sigma^{c}$ that can be mapped to $M$.

\subsection{The main result}

\section{Lemma 4.1}

Under shy ontologies, $D^{c} \cup \Sigma^{c} \models q^{c}$ if, and only if, $D^{c} \cup \Sigma^{c} \models_{\text {fin }} q^{c}$.

Proof

Clearly, the "only if" implication is straightforward. Hence, given a shy ontology $\Sigma$, we have to prove that $D^{c} \cup \Sigma^{c} \models q^{c}$, whenever $D^{c} \cup \Sigma^{c} \models_{\text {fin }} q^{c}$, for each database $D$ and UBCQ $q$. Suppose that $D^{c} \cup \Sigma^{c} \models_{\text {fin }} q^{c}$, i.e., the query $q^{c}$ is satisfied by each finite model of $D^{c} \cup \Sigma^{c}$. Thus, by Theorem 4.2, holds that $D^{c} \cup \Sigma^{c} \models_{\text {wsf }} q^{c}$, that is, the canonical rewriting of the query $q$ is satisfied by each well-supported finite model of the logical theory $D^{c} \cup \Sigma^{c}$. Then, by Theorem 4.3 , holds that $D^{c} \cup \Sigma_{a}^{c} \models_{\text {wsf }} q^{c}$, that is, the canonical rewriting of the query $q$ is satisfied by each wellsupported finite model of the joinless logical theory $D^{c} \cup \Sigma_{a}^{c}$. Moreover, again, by Theorem 4.2 we obtain that $D^{c} \cup \Sigma_{a}^{c} \models_{\text {fin }} q^{c}$, that is $q^{c}$ is satisfied also by every finite model of the previous theory. Now, as $\Sigma_{a}^{c}$ is a joinless ontology, by the finite controllability of joinless ontologies proved by Gogacz and Marcinkowski (2013), holds that $D^{c} \cup \Sigma_{a}^{c} \models q^{c}$. Finally, by Theorem 4.1 we have that $D^{c} \cup \Sigma^{c} \models q^{c}$, i.e. the query $q^{c}$ is satisfied by each model (finite or infinite) of $D^{c} \cup \Sigma^{c}$.

Summing-up, Theorem 1.1 follows by combining Lemma 4.1 with the properties of the canonical rewriting proved in Section 3 .

\section{Related work}

To complete the related works started with the Introduction, we recall that the notion of finite controllability was formalized for the first time by Rosati (2006) while he was working on a question that had been left open two decades before by Johnson and Klug (1984) about containment of conjunctive queries in case of both arbitrary and finite databases. Basically, using our terminology, they proved that ontologies mixing both inclusion-dependencies and functionaldependencies are not finitely controllable, by leaving open the case where ontologies contain inclusion-dependencies only. Rosati then answered positively this question.

The semantic equivalence of fundamental reasoning tasks under finite and infinite models is not at all a prerogative of the database community. A sister yet orthogonal property of finite controllability is of paramount importance also in logic, where it has been investigated much earlier. It is known as finite model property or finite satisfiability (Ebbinghaus and Flum 1995), and it asks for a class $\mathcal{C}$ of sentences whether every satisfiable sentence of $\mathcal{C}$ has a finite model. For example, both Gödel and Schütte proved that $\forall^{2} \exists^{*}$ first-order sentences are finitely satisfiable. 
Although reasoning under finite models has a long history and it has been actively investigated in various fields of Computer Science, finite controllability remains open for many languages combining or generalizing the key properties underlying the basic classes depicted in Figure 1] By way of example, we mention $(i)$ glut-guarded (Krötzsch and Rudolph 2011), extending guarded and weakly-acyclic; (ii) weakly-sticky-join (Calì et al. 2012), extending stickyjoin, weakly-acyclic and shy; and (iii) tame (Gottlob et al. 2013), extending sticky and guarded.

Between guarded and glut-guarded, it is worth to recall weakly-guarded (Calì et al. 2013), where each rule body has an atom covering all those variables that only occur in invaded (a.k.a. affected) positions. Actually, this class is finitely controllable although the proof sketch given by Bárány et al. (2014) has some hole (there, some model of $D \cup \Sigma^{\prime}$ might not satisfy $\Sigma$ ). In fact, our canonical rewriting yields an ontology that can be partitioned in active and harmless, where the active part is guarded. Well-supported models and propagation ordering behave as for shy.

An additional clarification concerns the notions of linear and sticky-join considered by Gogacz and Marcinkowski (2017), since they are not standard (actually stricter). In the former, repeated variables are admitted only in rule heads, while for the latter the authors state that the difference between sticky and stickyjoin "can only be seen if repeated variables in the heads of the rules are allowed". (Regarding sticky, the classical notion is only rephrased: their "immortal" positions correspond to positions being not marked.) From such a mismatch, however, it follows that finite controllability of stickyjoin was unknown before our work. A curious reader may verify that the proof of their Lemma 4 breaks down when moving to a linear (hence sticky-join) ontology such as $\Sigma=\{p(X, X) \rightarrow r(X)$; $r(X) \rightarrow \exists Y r(Y)\}$ —inducing no immortal position since all positions $p[1], p[2]$ and $r[1]$ host marked variables - paired with the singleton database $D=\{p(c, c)\}$.

\section{Conclusion}

By demonstrating that shy is finitely controllable, we complete an important picture around the basic decidable Datalog ${ }^{ \pm}$classes. But we take it as a starting point rather than an ending one.

On the one hand, finite controllability immediately implies decidability of OBQA. Actually, via the soundness and completeness of the chase procedure we know that the problem of deciding whether a UBCQ is true over a Datalog ${ }^{ \pm}$theory is recursively enumerable. But the complementary problem of deciding whether a UBCQ is false over a finitely controllable Datalog ${ }^{ \pm}$class $\mathcal{C}$ is recursively enumerable too. In fact, each theory $D \cup \Sigma$, with $\Sigma \in \mathcal{C}$, always admits a fair lexicographic enumeration of its finite models. Unfortunately, such a naïve procedure would be inefficient in practice. Making it usable and competitive for real world problems is challenging and it is part of our ongoing work. Basically, this would lead to a tool able to deal with any finitely controllable fragment, some of which (e.g., guarded) have no effective implementation.

On the other hand, we believe the techniques developed in this paper could have future applications. For example, we are working on an extended version of our canonical rewriting that encodes in the predicates also a limited amount of nulls. This requires more complex techniques, which however would apply to classes using the key properties underlying weakly-acyclic, such as glut-guarded and weakly-sticky-join (see Section 5). Hence, by combining these techniques with the above tool for finitely controllable classes, we aim at the design and implementation of a reasoner able to deal with ontologies falling in any known decidable Datalog ${ }^{ \pm}$class.

Finally - even if the unrestricted set of existential rules cannot be finitely controllable since it is not decidable - it is still open, to the best of our knowledge, whether there exists, or not, a fragment of existential rules which is decidable but not finitely controllable. 


\section{Acknowledgement}

The paper has been partially supported by the Italian Ministry for Economic Development (MISE) under project "PIUCultura - Paradigmi Innovativi per l'Utilizzo della Cultura" (n. F/020016/0102/X27), and under project "Smarter Solutions in the Big Data World (S2BDW)" (n. F/050389/0103/X32) funded within the call "HORIZON2020” PON I\&C 2014-2020.

\section{References}

Batder, F., Calvanese, D., McGuinness, D. L., Nardi, D., And Patel-Schneider, P. F., Eds. 2003. The Description Logic Handbook: Theory, Implementation, and Applications. CUP.

BAget, J., LeClÈre, M., AND Mugnier, M. 2010. Walking the decidability line for rules with existential variables. In Proc. of KR'10.

Baget, J., Leclère, M., Mugnier, M., And Salvat, E. 2009. Extending decidable cases for rules with existential variables. In Proc. of IJCAI'09. 677-682.

Baget, J., Leclère, M., Mugnier, M., And Salvat, E. 2011. On rules with existential variables: Walking the decidability line. AIJ 175, 9-10, 1620-1654.

BÁrÁny, V., Gottlob, G., And Отto, M. 2014. Querying the guarded fragment. Logical Methods in Computer Science 10, 2.

Bienvenu, M., ten Cate, B., Lutz, C., And Wolter, F. 2014. Ontology-based data access: A study through disjunctive datalog, csp, and MMSNP. ACM TODS 39, 4, 33:1-33:44.

Bourhis, P., Manna, M., Morak, M., And PIERIS, A. 2016. Guarded-based disjunctive tuplegenerating dependencies. ACM TODS 41, 4, 27:1-27:45.

Calì, A., Gottlob, G., AND KIfER, M. 2013. Taming the infinite chase: Query answering under expressive relational constraints. J. Artif. Intell. Res. (JAIR) 48, 115-174.

Calì, A., Gottlob, G., And Lukasiewicz, T. 2009a. Datalog ${ }^{ \pm}$: a unified approach to ontologies and integrity constraints. In Proc. of ICDT'09. 14-30.

Calì, A., Gottlob, G., And LukAsiewicz, T. 2009b. Tractable query answering over ontologies with datalog+/-. In Proc. of DL'09.

Calì, A., Gottlob, G., And Lukasiewicz, T. 2012. A general datalog-based framework for tractable query answering over ontologies. J. Web Sem. 14, 57-83.

CAlì, A., Gottlob, G., AND PIERIS, A. 2010. Advanced processing for ontological queries. PVLDB 3, 1, 554-565.

Calì, A., Gottlob, G., AND PIERIS, A. 2012. Towards more expressive ontology languages: The query answering problem. AIJ 193, 87-128.

Calvanese, D., De Giacomo, G., Lembo, D., Lenzerini, M., And Rosati, R. 2013. Data complexity of query answering in description logics. AIJ 195, 335-360.

Civili, C. And Rosati, R. 2012. A broad class of first-order rewritable tuple-generating dependencies. In Proc. of Datalog 2.0. 68-80.

Deutsch, A., NAsh, A., And Remmel, J. B. 2008. The chase revisited. In Proc. of PODS'08. 149-158.

Ebbinghaus, H.-D. And Flum, J. 1995. Satisfiability in the Finite. Springer Berlin Heidelberg, 95-103.

FAGES, F. 1991. A new fixpoint semantics for general logic programs compared with the well-founded and the stable model semantics. New Generation Comput. 9, 3/4, 425-444.

Fagin, R., Kolaitis, P. G., Miller, R. J., And PoPa, L. 2005. Data exchange: semantics and query answering. TCS 336, 1, 89-124.

Gogacz, T. And Marcinkowski, J. 2013. Converging to the chase - A tool for finite controllability. In Proc. of LICS'13. 540-549.

Gogacz, T. And Marcinkowski, J. 2017. Converging to the chase - A tool for finite controllability. JCSS 83, 1, 180-206. 
Gottlob, G., Kikot, S., Kontchakov, R., Podolskit, V. V., Schwentick, T., And ZAKHARYASCHEV, M. 2014. The price of query rewriting in ontology-based data access. AIJ 213, 42-59.

Gottlob, G., Manna, M., And Pieris, A. 2013. Combining decidability paradigms for existential rules. TPLP 13, 4-5, 877-892.

Gottlob, G., Orsi, G., AND Pieris, A. 2014. Query rewriting and optimization for ontological databases. ACM TODS 39, 3, 25:1-25:46.

Gottlob, G., PIERIS, A., And Tendera, L. 2013. Querying the guarded fragment with transitivity. In Proc. of ICALP'13. 287-298.

Johnson, D. S. AND KLUG, A. C. 1984. Testing containment of conjunctive queries under functional and inclusion dependencies. JCSS 28, 1, 167-189.

KRÖTZSCH, M. AND RUdOLPH, S. 2011. Extending decidable existential rules by joining acyclicity and guardedness. In Proc. of IJCAI'11. 963-968.

Leone, N., Manna, M., Terracina, G., And Veltri, P. 2012. Efficiently computable Datalog ${ }^{\exists}$ programs. In Proc. of KR'12.

PÉrez-Urbina, H., Motik, B., AND Horrocks, I. 2010. Tractable query answering and rewriting under description logic constraints. J APPL LOGIC.

Rosati, R. 2006. On the decidability and finite controllability of query processing in databases with incomplete information. In Proc. of PODS'06.

Ros ATI, R. 2007. The limits of querying ontologies. In Proc. of ICDT'07. 164-178. 


\section{Appendix}

\section{Shy existential rules}

This section is devoted to recall the formal definition of shy ontologies and their syntactic properties, as defined in Leone et al. (2012). For notational convenience and without loss of generality, we assume here that each pair of rules of an ontology share no variable. Let $\Sigma$ be an ontology, $\alpha$ be a $m$-arity atom, $i \in\{1, \ldots, m\}$ be an index, $\operatorname{pred}(\alpha)=a$, and $X$ be an existential variable occurring in some rule of $\Sigma$. We say that position $a[i]$ is invaded by $X$ if there exists a rule $\rho \in \Sigma$ such that $\operatorname{head}(\rho)=\alpha$ and

(i) $\alpha[i]=X$; or

(ii) $\alpha[i]$ is a universal variable of $\rho$ and all of its occurrences in $\operatorname{body}(\rho)$ appear in positions invaded by $X$.

Let $\phi(\mathbf{X})$ be a conjunction of atoms, and let $X \in \mathbf{X}$. We say that $X$ is attacked by a variable $Y$ in $\phi(\mathbf{X})$ if all the positions where $X$ appears are invaded by $Y$. On the other hand, we say that $X$ is protected in $\phi(\mathbf{X})$, if it is attacked by no variable.

A rule $\rho$ of an ontology $\Sigma$ is called shy w.r.t. $\Sigma$ if the following conditions are both satisfied:

(i) if a variable $X$ occurs in more than one body atom, then $X$ is protected in $\operatorname{bod} y(\rho)$;

(ii) if two distinct variables are not protected in $\operatorname{body}(\rho)$ but occur both in head $(\rho)$ and in two different body atoms, then they are not attacked by the same variable.

Finally, if each $\rho \in \Sigma$ is shy w.r.t. $\Sigma$, then call $\Sigma$ a shy ontology.

\section{Example 6.1}

Consider the following rules

$$
\begin{array}{rlll}
\rho_{1}= & s\left(X_{1}\right) & \rightarrow & \exists Y_{1} p\left(X_{1}, Y_{1}\right) ; \\
\rho_{2}= & p\left(X_{2}, Y_{2}\right), u\left(Y_{2}\right) & \rightarrow & r\left(X_{2}, Y_{2}\right) ; \\
\rho_{3}= & t\left(X_{3}\right) & \rightarrow & \exists Y_{3} u\left(Y_{3}\right) .
\end{array}
$$

Let $\Sigma=\left\{\rho_{1}, \rho_{2}, \rho_{3}\right\}$. Clearly, $\rho_{1}$ and $\rho_{3}$ are shy rules w.r.t. $\Sigma$, since they are also linear rules, namely rules with one single body atom, which cannot violate any of the two shy conditions. Moreover, rule $\rho_{2}$ is also shy w.r.t. $\Sigma$ as the positions $p[2]$ and $u[1]$ are invaded by disjoint sets of existential variables. Indeed, $p[2]$ is invaded by the existential variable $Y_{1}$ of the first rule, and $u[1]$ is invaded by the existential variable $Y_{3}$ of the third rule. Therefore, $\Sigma$ is a shy ontology.

Now, consider the further three existential rules

$$
\begin{array}{rlll}
\rho_{4}= & u\left(X_{4}\right) & \rightarrow & \exists Y_{4} p\left(Y_{4}, X_{4}\right) ; \\
\rho_{5}= & u\left(X_{5}\right) & \rightarrow & \exists Y_{5} p\left(X_{5}, Y_{5}\right) ; \\
\rho_{6}= & r\left(X_{6}, X_{6}\right) & \rightarrow & v\left(X_{6}\right) .
\end{array}
$$

Let $\Sigma^{\prime}$ be the ontology $\Sigma \cup\left\{\rho_{4}\right\}$. It is easy to see that $\rho_{1}, \rho_{3}$ and $\rho_{4}$ are shy w.r.t. $\Sigma^{\prime}$. However, $\rho_{2}$ is not shy w.r.t. $\Sigma^{\prime}$, as property $(i)$ is not satisfied. Indeed, the variable $Y_{2}$ occurring in two body atoms in $\operatorname{bod} y\left(\rho_{2}\right)$ is not protected, as the position $p[2]$ and $u[1]$ (the only positions in which $Y_{2}$ occurs) are invaded by the same existential variable, namely $Y_{3}$. Therefore, $\Sigma^{\prime}$ is not a shy ontology.

Let $\Sigma^{\prime \prime}$ be the ontology $\Sigma \cup\left\{\rho_{5}, \rho_{6}\right\}$. Again, $\rho_{1}, \rho_{3}, \rho_{5}$ and $\rho_{6}$ are trivially shy w.r.t. $\Sigma^{\prime \prime}$; and again $\rho_{2}$ is not shy w.r.t. $\Sigma^{\prime \prime}$. However, this time, $\rho_{2}$ is not shy because property (ii) is not satisfied. Indeed, the universal variables $X_{2}$ and $Y_{2}$, occurring in two different body atoms and in 
head $\left(\rho_{2}\right)$, are not protected in $\operatorname{bod} y\left(\rho_{2}\right)$, as the position $p[1]$ and $u[1]$ (in which occur $X_{2}$ and $Y_{2}$, respectively) are attacked by the same variable $Y_{3}$. Therefore, $\Sigma^{\prime \prime}$ is not a shy ontology.

Essentially, during every possible chase step, condition $(i)$ guarantees that each variable that occurs in more than one body atom is always mapped into a constant. Although this is the key property behind shy, we now explain the role played by condition $(i i)$ and its importance. To this aim, we exploit again $\Sigma^{\prime \prime}$, as introduced in the previous example, and we reveal why this second condition, in a sense, turns into the first one. Indeed, the rule $\rho_{6}$ bypasses the propagation of the same null in $\rho_{2}$ via different variables. However, one can observe that the rules $\rho_{2}$ and $\rho_{6}$ imply the rule $\rho_{6}^{\prime}: p\left(X_{6}, Y_{6}\right), u\left(X_{6}\right) \rightarrow v\left(X_{6}\right)$, which of course does not satisfy condition $(i)$. Actually, it is not difficult to see that every ontology can be rewritten (independently from $D$ and $q$ ) into an en equivalent one (w.r.t. query answering) where all the rules satisfy condition $(i)$. As an example, consider the following rule $\rho$

$$
p\left(X_{1}, Y_{1}\right), r\left(Y_{1}, Z_{1}\right), u\left(Z_{1}, Y_{1}\right) \quad \rightarrow \quad \exists W_{1} t\left(X_{1}, Z_{1}, W_{1}\right),
$$

and assume that it belongs to some ontology $\Sigma$ and that it is not shy w.r.t. $\Sigma$ because it violates condition $(i)$ only. Let us now construct $\Sigma^{\prime}$ as $\Sigma \backslash\{\rho\}$ plus the following two rules:

$$
\begin{aligned}
p\left(X_{1}, Y_{1}\right), r\left(Y_{1}^{\prime}, Z_{1}\right), u\left(Z_{1}^{\prime}, Y_{1}^{\prime \prime}\right) & \rightarrow \operatorname{aux}_{\rho}\left(X_{1}, Y_{1}, Y_{1}^{\prime}, Z_{1}, Z_{1}^{\prime}, Y_{1}^{\prime \prime}\right) ; \\
\operatorname{aux}_{\rho}\left(X_{1}, Y_{1}, Y_{1}, Z_{1}, Z_{1}, Y_{1}\right) & \rightarrow \exists W_{1} t\left(X_{1}, Z_{1}, W_{1}\right) .
\end{aligned}
$$

Both the new rules satisfy now condition $(i)$ w.r.t. $\Sigma^{\prime}$. Moreover, it is not difficult to see that, for every database $D$ and for every UBCQ $q$, it holds that $D \cup \Sigma \models q$ if and only if $D \cup \Sigma^{\prime} \models q$. However, since $\rho$ does not satisfy condition $(i)$, this immediately implies that the first new rule does not satisfy condition (ii).

The syntactic properties of shy make the class quite expressive since it strictly contains both linear and datalog. Moreover, these properties are easy recognizable and guarantee efficient answering to conjunctive queries, as experimentally shown in Leone et al. (2012). In fact, ontologybased query answering over shy ontologies preservers the same data and combined complexity of OBQA over datalog, namely PTIME-complete and EXPTIME-complete, respectively.

\section{Formal Proofs}

\section{Proof of Proposition 3.2}

We prove that $\Re\left(\operatorname{chase}\left(D^{c}, \Sigma^{c}\right)\right)=\operatorname{chase}(D, \Sigma)$ by induction on the chase step. Let $I_{0}=D \subset I_{1} \subset$ $\ldots \subset I_{m} \subset \ldots$ be a chase procedure of $D$ and $\Sigma$; and let $I_{0}^{c}=D^{c} \subset I_{1}^{c} \subset \ldots \subset I_{m}^{c} \subset \ldots$ be a chase procedure of $D^{c}$ and $\Sigma^{c}$.

Clearly, the base case follows, since, by definition of the canonical rewriting of $D, \mathfrak{R}\left(D^{c}\right)=D$.

Then, assume that $\Re\left(I_{m}^{c}\right)=I_{m}$. We have to prove that $\Re\left(I_{m+1}^{c}\right)=I_{m+1}$. By definition of chase step, there exist a rule $\rho \in \Sigma$ and a homomorphism $h$ from $\operatorname{body}(\rho)$ to $I_{m}$, such that $\langle\rho, h\rangle\left(I_{m}\right)=$ $I_{m+1}$. That is, $I_{m+1}=I_{m} \cup\{h(h e a d(\rho))\}$. By construction of a canonical rule, there exists a safe substitution $\varsigma$ w.r.t. $\rho$, such that $\varsigma(\rho)^{c}$ is a canonical rule and, by inductive hypothesis, there exists a homomorphism $h^{c}$ from body $\left(\varsigma(\rho)^{c}\right)$ to $I_{m}^{c}$. Consider the following homomorphism $\left(h^{c}\right)^{\prime}=(h \backslash h \mid \mathbf{X}) \cup h^{c}\left|\mathbf{x} \supseteq h^{c}\right| \mathbf{x}$. Therefore, $I_{m+1}^{c}=I_{m}^{c} \cup\left\{\left(h^{c}\right)^{\prime}(h e a d(\langle\rho, \varsigma\rangle))\right\}$. Moreover,

$$
\begin{aligned}
\mathfrak{R}\left(I_{m+1}^{c}\right) & =\mathfrak{R}\left(I_{m}^{c} \cup\left\{\left(h^{c}\right)^{\prime}\left(\text { head }\left(\varsigma(\rho)^{c}\right)\right)\right\}\right) & & = \\
& =\mathfrak{R}\left(I_{m}^{c}\right) \cup \mathfrak{R}\left(\left\{\left(h^{c}\right)^{\prime}\left(\text { head }\left(\varsigma(\rho)^{c}\right)\right)\right\}\right) & & = \\
& =I_{m} \cup\left\{h^{\prime}\left(\mathfrak{R}\left(\text { head }\left(\varsigma(\rho)^{c}\right)\right)\right)\right\} & & = \\
& =I_{m} \cup\left\{h^{\prime}(\text { head }(\rho))\right\} & & =I_{m+1} .
\end{aligned}
$$


Finally, let $q^{c}$ be the canonical rewriting of the UBCQ $q=\exists \mathbf{Y}_{1} \psi_{1}\left(\mathbf{Y}_{1}\right) \vee \ldots \vee \exists \mathbf{Y}_{k} \psi_{k}\left(\mathbf{Y}_{k}\right)$. For each $j \in\{1, \ldots, k\}$, consider the safe substitution $\varsigma_{j}$ mapping each variable of $\psi_{j}\left(\mathbf{Y}_{j}\right)$ in a different null. Therefore, there exists a conjunction of atoms, say $\psi_{j}^{c}\left(\mathbf{Y}_{j}\right)=\varsigma_{j}\left(\psi_{j}\left(\mathbf{Y}_{j}\right)\right)^{c}$ in $q^{c}$, such that $\mathfrak{R}\left(\psi_{j}^{c}\left(\mathbf{Y}_{j}\right)\right)=\psi_{j}\left(\mathbf{Y}_{j}\right)$, for each $j \in\{1, \ldots, k\}$. Hence, $q \subseteq \mathfrak{R}\left(q^{c}\right)$. Moreover, it is easy to see that, each other safe substitution $\varsigma^{\prime}$ w.r.t. some $\psi_{j}$, produces a conjunction of atoms, $\varsigma^{\prime}\left(\psi_{j}\left(\mathbf{Y}_{j}\right)\right)^{c}$ such that $\mathfrak{R}\left(\varsigma^{\prime}\left(\psi_{j}\left(\mathbf{Y}_{j}\right)\right)^{c}\right)$ is contained in $\mathfrak{R}\left(\varsigma_{j}\left(\psi_{j}\left(\mathbf{Y}_{j}\right)\right)^{c}\right)$. Therefore, $\mathfrak{R}\left(q^{c}\right) \subseteq q$. Thus, $\mathfrak{R}\left(q^{c}\right)=q$.

\section{Proof of Theorem 3.1}

We know that, for each database $D$, ontology $\Sigma$ and UBCQ $q$, it holds that $D \cup \Sigma \models q$ if and only if $\operatorname{chase}(D, \Sigma) \models q$ (Fagin et al. 2005). Therefore, also $D^{c} \cup \Sigma^{c} \models q^{c}$ if and only if chase $\left(D^{c}, \Sigma^{c}\right) \models$ $q^{c}$. Moreover, by Proposition 3.2, we have that $\mathfrak{R}\left(\operatorname{chase}\left(D^{c}, \Sigma^{c}\right)\right)=\operatorname{chase}(D, \Sigma)$ and $\mathfrak{R}\left(q^{c}\right) \equiv q$. Hence, remain to prove that $\mathfrak{R}\left(\operatorname{chase}\left(D^{c}, \Sigma^{c}\right)\right) \models \Re\left(q^{c}\right)$ if and only if $\operatorname{chase}\left(D^{c}, \Sigma^{c}\right) \models q^{c}$.

We prove the "if" part, given that the "only if" part can be obtained retracing the chain of the following implications. Suppose that $\operatorname{chase}\left(D^{c}, \Sigma^{c}\right) \models q^{c}$. Therefore, there is a homomorphism $h$ from at least one disjunct of $q^{c}$, say $\varsigma_{j}\left(\psi_{j}\left(\mathbf{Y}_{j}\right)\right)^{c}$ (where $\varsigma_{j}$ is a canonical substitution), to chase $\left(D^{c}, \Sigma^{c}\right)$, that is $h\left(\varsigma_{j}\left(\psi_{j}\left(\mathbf{Y}_{j}\right)\right)^{c}\right) \subseteq \operatorname{chase}\left(D^{c}, \Sigma^{c}\right)$. Therefore, $\mathfrak{R}\left(h\left(\varsigma_{j}\left(\psi_{j}\left(\mathbf{Y}_{j}\right)\right)^{c}\right)\right)$ $\subseteq \mathfrak{R}\left(\right.$ chase $\left.\left(D^{c}, \Sigma^{c}\right)\right)$. Moreover, note that $\mathfrak{R}\left(h\left(\varsigma_{j}\left(\psi_{j}\left(\mathbf{Y}_{j}\right)\right)^{c}\right)\right)=h\left(\Re\left(\varsigma_{j}\left(\psi_{j}\left(\mathbf{Y}_{j}\right)\right)^{c}\right)\right)$. Hence, $h\left(\Re\left(\varsigma_{j}\left(\psi_{j}\left(\mathbf{Y}_{j}\right)\right)^{c}\right)\right) \subseteq \mathfrak{R}\left(\operatorname{chase}\left(D^{c}, \Sigma^{c}\right)\right)$. Thus, $h$ is also a homomorphism from a disjunct of $\mathfrak{R}\left(q^{c}\right)$ to $\mathfrak{R}\left(\operatorname{chase}\left(D^{c}, \Sigma^{c}\right)\right)$, that is $\mathfrak{R}\left(\operatorname{chase}\left(D^{c}, \Sigma^{c}\right)\right) \models \mathfrak{R}\left(q^{c}\right)$.

\section{Proof of Proposition 4.1}

Let $\Sigma$ be a shy ontology. Note that, for each rule $\rho \in \Sigma$, there exists a rule $\varsigma(\rho)^{c} \in \Sigma^{c}$ such that $\varsigma\left(X^{i}\right)=n_{i}$ for each variable $X^{i}$ occurring in $\rho$. It is easy to see that a such $\varsigma$ is a safe substitution. We denote by $\bar{\Sigma}^{c}$ the set of all and anly this kind of rules in $\Sigma^{c}$. Note that, if $\Sigma^{c}$ is a shy ontology, then $\bar{\Sigma}^{c} \subseteq \Sigma^{c}$ is also a shy ontology.

By contradiction, suppose that $\bar{\Sigma}^{c}$ is not a shy ontology.

First, suppose that there exists a rule $\varsigma(\rho)^{c} \in \bar{\Sigma}^{c}$ such that there exists a variable, say $X$, occurring in more than one body atom and $X$ is not protected in $\operatorname{body}\left(\varsigma(\rho)^{c}\right)$. Therefore, for each existential variable $Y$, there exists an atom $\beta \in \operatorname{body}\left(\varsigma(\rho)^{c}\right)$ and some position $\operatorname{pred}(\beta)[i]$ in which $X$ occurs, and $\operatorname{pred}(\beta)[i]$ is not invaded by $Y$. Consider the unpacked rule $\mathfrak{R}\left(\varsigma(\rho)^{c}\right)=$ $\rho \in \Sigma$. Therefore, by construction, for each existential variable $Y$, there exists $\alpha \in \operatorname{body}(\rho)$ and some position pred $(\alpha)[j]$ in which $X$ occurs, and $\operatorname{pred}(\alpha)[j]$ is not invaded by $Y$. Hence, $X$ occurs in more than one body atom of $\rho$ and $X$ is not protected in $\operatorname{bod} y(\rho)$. So that, $\rho$ is not a shy rule, and, thus, $\Sigma$ is not a shy ontology.

Then, suppose that there exists a rule $\varsigma(\rho)^{c} \in \bar{\Sigma}^{c}$ such that there are two distinct universal variables, say $X$ and $Y$, that are not protected in $\operatorname{body}\left(\varsigma(\rho)^{c}\right)$; occur in head $\left(\varsigma(\rho)^{c}\right)$; occur in two different body atoms; and they are attacked by the same variable. Therefore, there exists an existential variable $Z$ such that $X$ and $Y$ occur only in invaded position by $Z$. Consider again the unpacked rule $\mathfrak{R}\left(\varsigma(\rho)^{c}\right)=\rho \in \Sigma$. Then, by the unpacking function, $X$ and $Y$ are not protected in $\operatorname{body}(\rho)$, and they occur in head $(\rho)$, in two different body atoms, and only in invaded position by $Z$. Thus, they are attacked by the same variable. Therefore, also in this case, $\rho$ is not a shy rule. Hence, $\Sigma$ is not a shy ontology. 
Proof of Proposition 4.2

Let $M$ be a finite model of $D \cup \Sigma$. Clearly, if $M$ is a well-supported finite model of $D \cup \Sigma$, we are done. Therefore, suppose that $M$ is not a well-supported finite model of $D \cup \Sigma$. Let $\Omega_{1}=$ $\left(\alpha_{1}, \ldots, \alpha_{m}\right)$ be an ordering of the atoms of $M$. Hence, by assumption, there exists $\alpha \in M$ that is not a well-supported atom w.r.t. $\Omega_{1}$. Let $\alpha_{j_{1}}$ be the first atom in the ordering $\Omega_{1}$ that is not wellsupported. And consider a new ordering $\Omega_{2}=\left(\alpha_{1}, \ldots, \alpha_{j_{1}-1}, \alpha_{j_{1}+1}, \ldots, \alpha_{m}, \alpha_{j_{1}}\right)$, where $\alpha_{j_{1}}$ is shifted from the position $j_{1}$ to the position $n$. As $M \notin w s f m o d s(D, \Sigma)$, then $\Omega_{2}$ is not a wellsupported ordering of $M$. Moreover, the first $j_{1}-1$ atoms are well-supported w.r.t. $\Omega_{2}$. Therefore, let $\alpha_{j_{2}}$ be the first atom in the ordering $\Omega_{2}$ that is not well-supported. Again, we consider a new ordering, say $\Omega_{3}$, where $\alpha_{j_{2}}$ is shifted from position $j_{2}-1$ to the position $n$. Iteratively, we build a sequence $\Omega_{1}, \Omega_{2}, \ldots, \Omega_{m}, \ldots$ of orderings that are not well-supported. Note that, as the number of different orderings is finite, there exist at least two orderings in the sequence that are the same. Therefore, let $\Omega_{m_{1}}$ and $\Omega_{m_{2}}$ be the first two orderings of the sequence, with $m_{2}>m_{1}$, such that $\Omega_{m_{1}}=\Omega_{m_{2}}$ (i.e., $\Omega_{m_{1}}$ and $\Omega_{m_{2}}$ are the same ordering). Consider the subset $A \subseteq M$ containing the first $n-\left(m_{2}-m_{1}\right)$ elements in $\Omega_{m_{1}}$, and the set $B$ of the last $m_{2}-m_{1}$ atoms in $\Omega_{m_{1}}$. By construction, $A$ is a well-supported instance. Moreover, each $\beta \in B$ is not well-supported by $A$, as $\Omega_{m_{2}}=\Omega_{m_{1}}$. That is, there is no rule $\rho$ in $\Sigma$ and no homomorphism $h$ such that $h(\operatorname{bod} y(\rho)) \subseteq A$ and $h(h e a d(\rho))=\{\beta\}$. Hence, as $M$ is a model, whenever $A \models \operatorname{bod} y(\rho)$, there exists an atom $\alpha$ in $A$, such that $\alpha \models$ head $(\rho)$. Therefore, $A$ is a model.

To complete the proof, let $M$ be a finite minimal model of $D \cup \Sigma$. As just proved, there exists a well-supported finite model $M^{\prime} \subseteq M$. By minimality of $M$, the model $M^{\prime}$ must be equal to $M$. Therefore, $M$ is a well-supported finite model.

Proof of Theorem 4.3

We have to prove that for each $M \in$ wsfmods $\left(D^{c}, \Sigma_{a}^{c}\right)$, there exist $M^{\prime} \in \operatorname{wsfmods}\left(D^{c}, \Sigma^{c}\right)$ and a homomorphism $h^{\prime}$ such that $h^{\prime}\left(M^{\prime}\right) \subseteq M$. Indeed, by hypothesis, there exists a homomorphism $h$ such that $h(q) \subseteq M^{\prime}$, and so $\left(h^{\prime} \circ h\right)(q) \subseteq M$.

Let $M \in$ wsfmods $\left(D^{c}, \Sigma_{a}^{c}\right)$, and let $\left(\alpha_{1}, \ldots, \alpha_{m}\right)$ be a well-supported ordering of $M$, and let $\left(\left\langle\alpha_{1}\right\rangle, \ldots,\left\langle\alpha_{m}\right\rangle\right)$ be a propagation ordering of $\left(\alpha_{1}, \ldots, \alpha_{m}\right)$. If there exists a join rule $\rho \in \Sigma^{c}$ satisfied by $M$ with a null or a constant $t$ in the join variables, then we consider the set of join atoms in the body of $\rho$ w.r.t. the term $t$, say $A \subseteq M$. First, we substitute a term $t$ of some $\alpha \in A$ in position $l$, with the corresponding term $\langle t, j, k\rangle$ of $\langle\alpha\rangle$, that can be considered as a fresh null. This new atom is denoted by $\alpha^{\prime}$, so that $\alpha^{\prime}[l]=\langle t, j, k\rangle$. Then, for each $\alpha_{i} \in M$ such that $\left\langle\alpha_{i}\right\rangle[l]=$ $\langle t, j, k\rangle$, for some position $l$, we set $\alpha_{i}^{\prime}[l]=\langle t, j, k\rangle$. Otherwise, $\alpha_{i}^{\prime}[l]=\alpha_{i}[l]$. In this way, we build an instance $M^{\prime}=\left\{\alpha^{\prime}: \alpha \in M\right\}$ of $\Sigma$, and a homomorphism $h^{\prime}$ such that $h^{\prime}(\langle t, j, k\rangle)=t$, for each introduced fresh null $\langle t, j, k\rangle$ to substitute $t$. By construction, it holds that $h^{\prime}\left(\alpha^{\prime}\right)=\alpha$, so that $h^{\prime}\left(M^{\prime}\right)=M$. Note that, by construction, $M^{\prime}$ is a well-supported finite instance of $D^{c} \cup \Sigma^{c}$.

Therefore, it remains to prove that $M^{\prime}$ is a model of $D^{c} \cup \Sigma^{c}$. By contradiction, suppose that $M^{\prime}$ is not a model. Hence, there exists a rule $\rho \in \Sigma^{c}$ such that $M^{\prime} \models \operatorname{body}(\rho)$, and $M^{\prime} \not \models h e a d(\rho)$. We distinguish two cases.

(i) First, suppose that $\rho$ is not a join rule. Then, there exists a safe substitution $\hat{\zeta}$, mapping each variable in the atoms of $\rho$ into a different null, so that $\hat{\zeta}(\rho)^{c} \in \Sigma_{a}^{c}$, as it is not a harmless rule of Shy. By hypothesis, $M^{\prime} \models \operatorname{body}(\rho)$, so that there exists a homomorphism $h^{\prime \prime}$ such that $h^{\prime \prime}(\operatorname{bod} y(\rho)) \subseteq M^{\prime}$. Therefore, $h^{\prime}\left(h^{\prime \prime}(\operatorname{bod} y(\rho))\right) \subseteq h^{\prime}\left(M^{\prime}\right)=M$, and so $M \models \operatorname{bod} y(\rho)$. Hence, also $M \models \operatorname{body}\left(\hat{\zeta}(\rho)^{c}\right)$. As $M$ is a model of $\Sigma_{a}^{c}$, then $M \models h e a d\left(\hat{\zeta}(\rho)^{c}\right)$. Therefore, there exists a homomorphism $h^{\prime \prime \prime}$ such that $h^{\prime \prime \prime}\left(\operatorname{head}\left(\hat{\zeta}(\rho)^{c}\right)\right)=\alpha_{j}$, for some $j \in\{1, \ldots, m\}$. Hence, 
$\alpha_{j} \in M$. Therefore, $\alpha_{j}^{\prime} \in M^{\prime}$. Moreover, $\alpha_{j}^{\prime} \models \operatorname{head}(\rho)$, as $h^{\prime}\left(\alpha_{j}^{\prime}\right)=\alpha_{j} \models \operatorname{head}(\rho)$. Therefore, $M^{\prime} \models \operatorname{head}(\rho)$.

(ii) Now, suppose that $\rho$ is a join rule. Since, by hypothesis, $M^{\prime} \models \operatorname{bod} y(\rho)$, then, the join variables in the body of $\rho$ are instantiated by the same null, as $D^{c} \cup \Sigma^{c}$ is a constant-free logical theory. However, by construction of $M$, it is not possible that the same term comes from an instantiation of two different existential variables, since we replaced each such instantiation with a fresh null in at least one joined term.

Therefore, $M^{\prime}$ is a well-supported finite model of $D^{c} \cup \Sigma^{c}$. 